\title{
Comparison of the mechanical behaviour of standard and auxetic foams by X-ray Computed Tomography and Digital Volume Correlation
}

\author{
F. Pierron ${ }^{1}$, S.A. McDonald ${ }^{2}$, D. Hollis ${ }^{3}$, J. Fu ${ }^{4,5}$, P.J. Withers ${ }^{2}$, A. \\ Alderson ${ }^{6}$
}

${ }^{1}$ Faculty of Engineering and the Environment, University of Southampton, Highfield, Southampton, SO17 1BJ, UK

${ }^{2}$ School of Materials, University of Manchester, Grosvenor St, Manchester, M1 7HS, UK

${ }^{3}$ LaVision UK Ltd, Downsview House, Grove Technology Park, Grove, Oxon, OX12 9FF, UK

${ }^{4}$ LMPF, Arts et Metiers ParisTech, Rue St-Dominique, 51000 Chalons-en-Champagne, France

${ }^{5}$ Wolfson School of Mechanical and Manufacturing Engineering, Loughborough University, Leicestershire, LE11 3TU, UK

${ }^{6}$ Materials and Engineering Research Institute, Faculty of ACES, Sheffield Hallam University, Howard Street, Sheffield, S1 1WB, UK

Keywords: X-ray CT, digital volume correlation, auxetic foam, low density polymeric foam, Poisson's ratio.

\begin{abstract}
The tensile behaviour of standard and auxetic polyurethane foams are contrasted by digital volume correlation (DVC) of 3D images collected by in situ X-ray computed tomography (CT). It was found that subset sizes of 32 and 64 voxels for the auxetic and standard foams were optimal for strain resolutions in the order of $0.1 \%$. For the standard foam good uniformity of strain was observed at low strains giving a tangent Poisson's ratio of 0.5 . Some heterogeneity of strain was observed at higher strains which may be related to the fixtures. The behaviour of the auxetic foam was totally different, with strain being spatially heterogeneous with transverse strains both positive and negative but giving a negative Poisson's ratio on average. This suggests that the unfolding tendency of some groups of cells was higher than others because of the complex frozen starting microstructure. Further different methods of deriving Poisson's ratio gave different results. Besides revealing interesting microstuctural mechanisms of transverse straining the study also shows DVC of tomography sequences to be the perfect tool to study complex mechanical behaviour of cellular materials.
\end{abstract}

\section{Introduction}

Low density polymeric foams are widely used in applications that require good low energy absorption capabilities, such as in packaging for instance. However, their mechanical characterization is a challenge because of their large deformation and their tendency for strain localization [1]. Many constitutive models have been devised to describe their mechanical behaviour but the experimental identification procedures still largely rely on standard uniaxial tests that are too limited to fully capture the complexity of their behaviour. Surface full-field deformation measurements such as digital image correlation enable one get more information about this behaviour in order to better extract intrinsic material properties such as tangent Poisson's ratio at different levels of 
compression, as detailed in [1]. However, surface measurements are not sufficient to completely address the complex 3D large deformation behaviour of such materials.

Measuring strains inside solids is a difficult task but different techniques have been developed over the years. Their field of application depends mainly on the material under consideration. There have been attempts at using embedded strain gauges $[2,3]$ but mostly, the techniques rely on non-contact volume imaging of deformation from a number of different physical information. In materials that exhibit an MRI signal (mainly biological materials with high water content), Magnetic Resonance Elastography provides high quality bulk deformation data [4-7]. Ultrasound-based techniques have also been used for biological tissues, as in [8,9], but with a lower resolution. When the materials are semi-transparent, interferometric techniques based on the scattering of light within the solids have been developed. A series of variants such as Phase-Contrast Spectral Optical Coherence Tomography (PC-SOCT, [10]), Tilt-Scanning Interferometry (TSI, [11]), Wavelength Scanning Interferometry (WSI, [12]) have been explored, which can all be seen as "a marriage between the phase sensing capabilities of Phase Shifting Interferometry and the depth-sensing capabilities of Optical Coherence Tomography" (quote from [13]). However, these methods are often too sensitive for a lot of applications and are hard to set-up and expensive, even though they provide excellent quality data, as seen in [11], for instance. Similar ideas using interferometry but with optical 'slicing' have been experimented in [14]. It should be noted that such techniques can be spoilt by the presence of strain induced light index variations (photo-elastic effect), which can however be accounted for [14]. Finally, neutron and synchrotron X-ray diffraction have also been widely used to map in the elastic strains at specific points, along specific lines or across 2D sections [15], but only elastic strains are measurable and for certain materials only.

Digital Volume Correlation (DVC) is becoming the most popular bulk strain measurement technique $[16,17]$. It is a $3 \mathrm{D}$ extension of the very well known Digital Image Correlation (DIC) technique [18]. A standard single camera DIC can measure in-plane deformations while using two cameras enable to measure the three components of the displacement field over a surface. DVC is a bulk extension of DIC where subsets of voxels containing some information contrast can be used to track the specimen deformation within the volume. Apart from the much longer computing times, the main difference between DVC and DIC is that for the latter, it is possible 
to artificially produce a pattern at the surface of the investigated solid by spraying paint for instance whereas for DVC, this is much more difficult. In order to perform DVC, one needs a digitized representation of the volume under investigation, with some random contrast patterns to allow for the correlation algorithm to be used successfully. This can be obtained by a number of techniques. First, incremental optical slicing can be employed, as reported in [19-21], but this restricted to semi-transparent materials. Also, scattering particles have to be embedded in the solid to improve the scattering contrast. The same sort of volume information can be obtained by Optical Coherence Tomography (OCT) which provides volume contrasts of optical index but the quality of the patterns is highly dependent on the tested material and as such, the strain resolutions can be very variable from one kind of specimen to the next [22]. Confocal microscopy was also used on samples which were seeded with fluorescent beads [23], as well as MRI [24]. Most of the studies reporting DVC results are based on X-ray computed tomographic (X-ray CT) volume reconstructions. X-ray CT involves reconstructing a volume of X-ray absorption contrast from a series of $2 \mathrm{D}$ images of this contrast acquired while rotating the sample [25, 26]. This technique is widely used to investigate the composition of solids, imaging heterogeneities within the bulk of material. The recent development of desktop micro-CT scanning systems has led to a spectacular increase in the use of CT scanning in materials science, but when a monochromatic beam is needed (say to reduce beam hardening artefacts) or when 3D images must be acquired over short timescales, synchrotron X-ray sources provide the best tool. Applications concern composites [27-29], granular materials [30,31], metals [32], foams [33,34], wood [35] etc.

Pioneering work on DVC dates back to 1999 in Bay's group [16,36,37] where it was developed to image the deformation of trabecular bone, for which the foam-like structure proved very well suited to DVC from X-ray CT. More recently, several groups worldwide have been involved in DVC development, see for instance [30,33,35,38-42]. Within the last two years, a commercial system has appeared on the market [43]. This turns DVC into an operational tool. This software package was used in the present study.

Auxetic (also known as negative Poisson's ratio, NPR) foams can be manufactured from low density conventional polyurethane foams by compression and heating, as described in [34]. There are several motivations for the development of such materials. For example, if a rigid auxetic foam is used in sandwich structures, it yields a constraining effect when impact compression is 
introduced on the sandwich skins, resulting in better damage tolerance [44], even though the lower stiffness of the auxetic foam remains an issue from a bending stiffness point of view. A second application for soft auxetic foams is for particle filtering, with the ability to vary the size of the pores by simple mechanical action [45]. For other possible applications of auxetic foams (and honeycomb structures), the reader is referred to [46]. Up to now, the mechanical behaviour of such materials has mainly been investigated at the macroscopic level [1,47] and at the surface, with the exception of $[34,48]$ where micro-CT combined with DIC on selected slices was performed. However, no attempt at full DVC analysis has been performed until now, to the best knowledge of the authors. However, the manufacturing process of such soft auxetic foams involves large compression of a standard foam resulting in buckling of the cells [48]. As a consequence, the auxetic foam has a shrivelled structure which turns it into a deployable material, like a crumpled sheet of paper. It is therefore questionable whether the macroscopic auxetic effect measured up till now is really representative of the local behaviour of cells or groups of cells in the auxetic foam. Possible heterogeneities would be of primary importance for the filtering application reported above for instance.

The objective of this work is to investigate the bulk deformation of auxetic foam specimens such as that in $[34,48]$, loaded in tension. These results will then be compared with similar data obtained on the precursor standard foam from which the auxetic foam has been manufactured. The purpose is to understand the possible specificities of the deformation patterns in the auxetic foam, and eventually, relate this to the manufacturing processes. In [34,48], standard surface digital image correlation was performed in slices but the quality was not good enough to look at the strain distributions. In the current paper, the materials and test conditions are first reported. Then, the metrological performances of the current DVC set-up is examined on stationary and rigid body translated reconstructed volumes, for both standard and auxetic foams. Results of tensile tests for both standard and auxetic foams are then examined and critically discussed. Finally, Poisson's ratio for both foams are derived and discussed. 


\section{Experimental procedure}

\subsection{Materials}

A low density polyurethane (PU) foam was used in the present study (Custom Foams, designated

by R45FR, 18 pores.cm ${ }^{-1}$, density of $30 \mathrm{~kg} \cdot \mathrm{m}^{-3}$ ). To convert the foam into an auxetic form, a cylinder of unconverted (standard) foam of $150 \mathrm{~mm}$ in length and $30 \mathrm{~mm}$ in diameter was inserted into a metallic tube of $20 \mathrm{~mm}$ circular internal diameter and $100 \mathrm{~mm}$ length. End tabs were then secured over the free ends of the mould to ensure compressive strain was applied along the mould axis. A linear compression ratio of 0.67 (the converted to unconverted dimensions) was thus applied, corresponding to a volumetric compression ratio of 0.3 . The mould and foam were then placed in an oven at a temperature of $190{ }^{\circ} \mathrm{C}$ for 15 minutes. The foam was removed and relaxed to avoid adhesion of ribs and to minimize surface creasing and then reinserted into the mould at $190{ }^{\circ} \mathrm{C}$ for a further 10 minutes, followed by 20 minutes at $100{ }^{\circ} \mathrm{C}$. The density of the resulting auxetic foam was determined to be $62 \mathrm{~kg} \cdot \mathrm{m}^{-3}$ ). In the currents study, both foams were tested: the standard and the auxetic one.

\subsection{Experimental set-up}

The specimens were tested in tension in the chamber of the X-ray imager using the fixture shown in Fig. 1. An essentially X-ray transparent polymeric cylinder was used to provide support for the wire used to extend the sample. For the auxetic specimen, two load steps were applied and three reconstructed volumes were recorded, volume A for the unloaded specimen, volume B at the end of the first load step and volume $\mathrm{C}$ at the end of the second. These are referred to as AA, AB, AC (see Table 1). For the standard foam, four volumes were recorded corresponding to three load steps, namely, SA, SB, SC and SD (see Table 1). In parallel, both auxetic and standard foam specimens were also used to evaluate the performances of the digital volume correlation for deformation measurements. In this case, three volumes were recorded. Volumes SA1 and SA2 are two consecutive reconstructions of the stationary auxetic specimen whereas TA corresponds to the specimen subjected to a vertical rigid body translation of about 30 voxels along direction z. The same procedure was also adopted for the standard foam specimen, see Table 1. 


\section{$2.3 \quad$ X-ray computed tomography}

X-ray microtomography measurements were carried out using a Nikon Xtek XTH 225 X-ray radiography/tomography set. Due to the cone beam X-ray source geometry of the tomography system the distance between the source and sample determined the magnification and thus the voxel size in the reconstruction. A region of interest (ROI) was scanned lying wholly within the sample dimensions, of size 10.5 by 10.5 by $10.1 \mathrm{~mm}^{3}$, such that an isotropic voxel size of $15 \mu \mathrm{m}$ was achieved in the reconstructed 3D volumes. An X-ray tube potential of $50 \mathrm{kV}$ (current $380 \mu A$ ) from a tungsten target was found to give sufficient contrast for the weakly absorbing polymeric foam material. The wavelength profile of the beam was shaped using a $0.1 \mathrm{~mm}$ aluminium filter placed just in front of the exit window of the $\mathrm{X}$-ray source. The projection data for each tomography scan comprised 721 radiographs, each $0.258^{\circ}$ apart, which were then reconstructed into 3D tomographical volumes using a cone-beam extension of the filtered backprojection algorithm. The exposure time for each radiograph was $1 \mathrm{~s}$, leading to total scan times around $12 \mathrm{mn}$ for one volume. No multiple radiograph averaging was performed. Reconstruction time was about $5 \mathrm{mn}$ for one volume. Typical slices of two reconstructed volumes, one for the standard foam and one for the auxetic foam, are given in Fig. 2. More slices of the reconstructed volumes can be found in the supplementary material, Videos 1 and 2. A 3D rendered view of both auxetic and standard foams can be found in Fig. 3. The wrinkled nature of the converted auxetic foam is clearly apparent there. From Fig. 3(b), one can see that the cell wall thickness is around 6 voxels, $i e, 0.1 \mathrm{~mm}$.

\subsection{Digital Volume Correlation}

Digital volume correlation was performed on the reconstructed tomographic volumes using the LaVision commercial package DaVis [43] based on a Fast Fourier Transform algorithm. Its main features are recalled below.

- The correlation is a local approach with each sub-volume pattern correlated independently.

- A multi-pass approach is used whereby large sub-volumes are initially used to capture large displacements. Subsequent to this, these initially calculated displacements are used to displace smaller sub-volumes, and thus ensure the pattern is followed and signal to noise 
ratio maximised.

- The shape function is piecewise linear.

- Gaussian curve-fitting of the correlation function peak is used to detect the position of the displacement with sub-voxel resolution.

Strains were calculated from centred finite difference ('gradient' function in Matlab ${ }^{\circledR}$ ), without any additional smoothing.

\section{Measurement performances}

A natural procedure to evaluate the displacement and strain resolutions of DVC is to analyze reconstructed volumes acquired under zero stress. This was performed on both auxetic and standard foams because of their different structures. The parameters of the X-ray tomography measurements can be found in Table 1. As is seen in Fig. 2, the density of the two foams is different (30 for the standard foam compared to $62 \mathrm{~kg} \cdot \mathrm{m}^{-1}$ for the auxetic one). The standard foam has 18 pores.cm ${ }^{-1}$, therefore the average pore size is slightly less than $1 \mathrm{~mm}$. With a voxel size of $15 \mu \mathrm{m}$, it was found that a subset size of $64 \times 64 \times 64$ voxel $^{3}$ was necessary to avoid lack of convergence of the correlation algorithm in certain subsets. Consequently, the subset length is $64 \times 15.10^{-3}=0.96 \mathrm{~mm}$, about the average size of the pores. This ensures that in such a volume, there will always be some cell wall material to provide the necessary contrast for the correlation algorithm. Following the same reasoning, a subset size of $48 \mathrm{x} 48 \mathrm{x} 48$ voxel $^{3}$ was used for the auxetic foam, providing a physical subset length of $48 \times 15.10^{-3}=0.72 \mathrm{~mm}$. Since the auxetic foam was obtained through a linear compression ratio of 0.67 , its average pore size is about $0.67 \mathrm{~mm}$ so again, the subset size is about the same size as the average pore, ensuring convergence of the correlation algorithm. The data are summarized in Table 2. Typical computing time for one load step was about $2 \mathrm{mn}$. Fig. 4 shows magnified $2 \mathrm{D}$ views of both foams, with a grid corresponding to 2D subset sizes, 48 for the auxetic foam and 64 for the standard one. Since the objective of this work is to compare the mechanical behaviour of both foams, it was found convenient to have a spatial resolution of the DVC measurements equal to the average pore size for both foams. It should be pointed out that the objective of the present study is not to optimize the performances of DVC but to give an idea of resolution for the current 
set-up in order to better analyze the significance of the data on the deformed specimens. As a consequence, a detailed account of how the performances evolve with subset size is not reported here. From the raw strain maps, an outlier removal routine was used to remove data points which were more than twice as large in magnitude as the standard deviation. This excluded outliers located primarily at the edges where the correlation data are noisier and heavily influenced by the masking procedure.

The first step in the evaluation of the DVC performances is to use two subsequent reconstructions of the same stationary volume, namely SA1 and SA2 for the auxetic foam, and SS1 and SS2 for the standard foam, see Table 1. From the obtained volume strain maps, the standard deviation of each strain component was calculated for each $(\mathrm{x}, \mathrm{y})$ slice and the values reported in Fig. 5. It should be noted that the mean of the strains (bias) is one order of magnitude lower than the standard deviation. On both plots, the standard deviation is constant along the different slices, with slight increase towards the ends, as is usual in image or volume correlation. The other common feature is that normal strains are noisier than shear strains, with the exception of $\varepsilon_{z z}$ for the standard foam. Finally, one can see that the noise levels are significantly larger for the standard foam (between 0.1 and $0.15 \%$ ) than for the auxetic foam (between $2.10^{-4}$ and $3.10^{-4}$. This is not surprising considering Fig. 2. Even though the subset size is larger for the standard foam, the lower density of this foam makes it more difficult to image for DVC purposes. And increasing the voxel size would not be an option as the cell walls still need to be resolved. In any case, these very flexible foams will deform to much larger levels of strain when tested in tension so these noise levels are acceptable for the current study. Fig. 6 shows the six strain components in a slice of the auxetic foam. As expected, one can see that the strain maps exhibit very little spatial correlation, maybe just that arising from the $50 \%$ overlap in DVC processing, which is satisfactory.

The second step to evaluate the strain resolution is to perform rigid body translation of the specimens. Indeed, the stationary reconstructions test the noise coming from the sensor, the environment (thermal stability of the X-ray CT chamber), the reconstruction algorithm etc., whereas a rigid body translation also puts to the test the interpolation features of the correlation algorithm, so larger strain standard deviations can be expected. These plots, obtained from the correlation of volume TA and SA1 for the auxetic foam, and TS and SS1 for the standard foam, 
are reported in Fig. 7. The same general conclusions as above can be drawn. The errors at both ends increase dramatically because of the z-translation. The translation is of about 30 voxel in the $\mathrm{z}$ direction, so as expected, between one and two data points are corrupted towards the end. One can also see a significant increase of the strain noise for the auxetic foam between stationary and translation tests, whereas it remains nearly constant for the standard foam, but at a much higher level. In the authors' opinion, this is consistent with the fact that for the standard foam, the strain error is mainly influenced by the contents of cell wall material in the subset (by analogy to 2D DIC, the number of 'speckles' in the subset) than by the interpolation function, whereas both error sources are mixed for the auxetic foam. This would need to be investigated further to reach a definite conclusion.

In conclusion to this section, it can be said that DVC was possible on $64^{3}$ and $48^{3}$ subsets for respectively standard and auxetic foams. Without any smoothing, strain resolutions lower than $6.10^{-4}$ were obtained for the auxetic foam while the strain resolution rises to about $0.15 \%$ for the standard foam, which is considered here as satisfactory compared to the strain levels that the specimens experience in the subsequent tensile tests.

\section{Results for the standard foam}

Each of the three load steps were analyzed using DVC with the procedure detailed previously. For the first load step, volume SA (undeformed) was used as reference and volume SB (deformed at load step 1) as the deformed stage. For load step 2, volume SB was used as reference (end of load step 1) and volume SC as the deformed stage. Finally, this was repeated for volumes SD ad SC, providing a set of three volume maps of incremental deformation from which strain was calculated without smoothing as for the noise study. The reason for choosing this incremental approach was that in doing so, one avoids the issue of significant change in the material structure because of deformation, that may affect the correlation quality. It was also natural in order to investigate the evolution of the average tangent Poisson's ratio, in the same spirit as in [1]. Finally, the main drawback of this procedure is the accumulation of noise at each step but this was not such an issue here as the strain levels are rather large compared to the strain resolution. The average $U_{z}$ displacement in $(x, y)$ slices has been plotted for all load steps as a function of $z$ in Fig. 8. For the three load steps, the average longitudinal displacement is linear with $z$, as 
expected. One can see that the strains are larger for load steps 2 and 3 compared to load step 1. This is confirmed in Fig. 9 which shows the average $\varepsilon_{z z}$ strain in $(x, y)$ slices as a function of $z$. The first load step produces lower strains than the two subsequent ones. The cumulated strain after load step 3 is about $4 \%$. Also, for some reason, the first two slices exhibit lower strains. This will have an impact on the Poisson's ratio results presented in Section 6.1. Videos of the normal strain fields through the volume are given in the supplementary material, for the first load step, Videos 3 to 8 . The last two slices were removed from the results, as explained in more details in Section 5 below. Typical strain maps are represented in Fig. 10, for one slice (here, slice 5) in load step 1 . The strain maps are reasonably uniform, with negative transverse strains indicating positive Poisson's ratios.

\section{Results for the auxetic foam}

Each of the two load steps were analyzed using DVC. For the first load step, volume AA (unloaded specimen) was used as reference and volume AB (deformed at load step 1) as the deformed stage. For load step 2, volume AB was used as reference (end of load step 1) and volume AC as the deformed stage. This provided two volume maps of incremental deformation from which strain was calculated without smoothing as in the noise study above. The average $U_{z}$ displacement in $(x, y)$ slices has been plotted for both load steps as a function of $z$ in Fig. 11. One can see that for both load steps, the average displacement is linear with $z$ up to about two thirds of the field of view. Then there is a change of slope as represented on the figure. One can also see that this slope change occurs further up in load step 2, which hints that this is a material related effect (as the material points have moved upwards after load step 1 due to the deformation). This suggests that the material is stiffer on the upper side of the field of view. This will be commented on later in the paper.

The six components of the strain field are represented as a sequence of $2 \mathrm{D}$ slices in the $(\mathrm{x}, \mathrm{y})$ plane, from the bottom to the top of the field of view. These results can be found as supplementary material in Section 8, Videos 9 to 14. It should be noted that the last two slices were removed from the results. Indeed, after load step one, the maximum displacement at the top of the specimen is about 30 voxel. Since the subset length is 48 pixel, and the shift is $50 \%$, then two data points at the top will be affected and need to be removed. Typical strain 
maps are represented in Fig. 12, for one slice (here, slice 13) in load step 1. The first comment from these maps is that they look very heterogeneous. $\varepsilon_{z z}$ has the largest strain amplitudes, as expected but there are very large variations within each slice. The same trend is observed on the other components. Of particular interest are the maps of $\varepsilon_{x x}$ and $\varepsilon_{y y}$. The foam is supposed to exhibit auxetic behaviour. Hence, these transverse strain components should be positive. The maps show while they are predominantly positive, there are patches of negative values, with very strong heterogeneity. The shear strain components also exhibit significant strain heterogeneities, as expected from the normal strain maps.

The next question that arises is whether or not these strain heterogeneities are 'real' and related to material behaviour. Fig. 13 brings an answer to that question. It shows the $\varepsilon_{y y}$ strain maps for the same material slice (slice 13) for both load steps. Since this slice is only about halfway up the field of view, it is subjected to a displacement corresponding to less than the size of one data point (24 voxel). So the indisputable spatial correlation between these two strain maps clearly shows that the measured heterogeneities are indeed related to the material behaviour and not to some measurement artefact. As a further proof, the figure also shows a typical strain map from the rigid translation test of Section 3, with the same scale as the map from load step 1. It is clear from this information that the heterogeneities are not produced by measurement noise as the amplitude of the strains is at least one order of magnitude larger than the noise levels.

Another interesting observation is that the heterogeneity patterns are different from one strain component to the next. Looking at $\varepsilon_{z z}$, one can see that the strain hotspots are not coincident with those in the $\varepsilon_{x x}$ and $\varepsilon_{y y}$ maps. The patterns in $\varepsilon_{z z}$ will be sensitive to the local variation of the Young modulus whereas hotspots for the transverse strain will arise from the mechanical unfolding effect which is the cause of the auxetic behaviour. It is not really surprising that these strain maps are not uniform when one considers the manufacturing process. A sample of standard foam is compressed in the three direction of space to a ratio of 0.3. Such compression will cause the cells to collapse elastically, but not necessarily in a uniform manner. As shown in [1], using surface DIC measurements in a unidirectional compression test, rows of cells gradually collapse until complete densification is reached. At a certain compressive strain before densification, the strain map is highly heterogeneous, with groups of cell completely collapsed and others not 
collapsed at all. If such a microstructure was frozen, then one would expect the collapsed cell to exhibit strong local unfolding power, whereas non collapsed cells would exhibit no (or very little) auxetic effect. This would lead to groups of highly auxetic cells 'pushing' their non or less auxetic neighbours and forcing them to compress, hence the positive and negative strains in the $\varepsilon_{x x}$ and $\varepsilon_{y y}$ maps.

In order to corroborate the above, the standard deviation of the six strain components has been plotted as a function of $z$ for load step 1, see Fig. 14. It is clear that all components exhibit a decrease of standard deviation around slice 18, exactly when the change of stiffness is apparent on Fig. 11. Fig. 14 also shows the standard deviation of $\varepsilon_{z z}$ for load step 2, which clearly exhibits the same trend (with larger values since the total axial strain is larger for load step 2). The discussion on this will be provided in the next section when Poisson's ratio is examined.

\section{Evaluation of Poisson's ratios}

The objective of this section is to complement the previous results by calculating and analysing Poisson's ratios for both standard and auxetic foams.

\subsection{Standard foam}

There are two natural ways to calculate Poisson's ratios. The first one involves constructing spatial maps of Poisson's ratios in each $z$ slice, and then averaging over each slice.

$$
\begin{aligned}
& \nu_{z x}^{1}=\overline{\left[-\frac{\varepsilon_{x x}}{\varepsilon_{z z}}\right]} \\
& \nu_{z y}^{1}=\overline{\left[-\frac{\varepsilon_{y y}}{\varepsilon_{z z}}\right]}
\end{aligned}
$$

where the over line denotes spatial averaging in the $(x, y)$ plane. The second method is based on averages of the axial and transverse strains within each slice:

$$
\begin{aligned}
& \nu_{z x}^{2}=-\frac{\overline{\varepsilon_{x x}}}{\overline{\varepsilon_{z z}}} \\
& \nu_{z y}^{2}=-\frac{\overline{\varepsilon_{y y}}}{\overline{\varepsilon_{z z}}}
\end{aligned}
$$

It should be noted that one could also average the strains over the whole volume instead of each $\mathrm{z}$ slice. The results in this case are very close to that provided by method 2. Fig. 15 
shows the tangent Poisson's ratios for the three load steps, obtained from both methods. The first conclusion is that both methods produce the same value for Poisson's ratio, as would be expected for a homogeneous material. Poisson's ratios are also positive for all load steps, with a tendency to consistently increase with the strain level towards 0.5. At load step 3, the $\nu_{z y}$ Poisson's ratio even crosses the 0.5 threshold. This may hints towards some load induced anisotropy while the cells are being stretched in one direction, but this would need to be confirmed. The very consistent results suggest however that this result is significant. Another surprising fact is the difference between $\nu_{z x}$ and $\nu_{z y}$ at load steps 2 and 3, whereas they are similar for load step 1 .

In order to investigate this, the average transverse strains $\varepsilon_{x x}$ and $\varepsilon_{y y}$ have been plotted as a function of $z$ in Fig. 16. It clearly appears that the magnitude of $\varepsilon_{y y}$ is much higher than that of $\varepsilon_{x x}$ for load steps 2 and 3, while identical for load step 1. It is not clear why this is happening. It might have been because of some parasitic non-uniaxial loading being introduced but typical strain maps for load step 2 plotted in Fig. 17 do not show any evidence of out-of-plane bending, for instance. This particular behaviour remains unexplained sofar and will need more thorough investigation in future work.

\subsection{Auxetic foam}

Maps of Poisson's ratio calculated with the first method described above are provided in Videos 15 and 16 for load step 1 . One can clearly see the strong heterogeneity of these maps following the heterogeneities in Fig. 13. The patterns tend to follow that of the associated transverse strain, as can be seen on Fig. 18. One can also see an increase with slice number of the positive strain amplitude over the surfaces of the slices exhibiting auxetic effect.

Fig. 19(a) shows the evolution of both Poisson's ratios as a function of $z$, for both methods presented above. Interestingly, both methods provide rather different results, even though the trend of increasing magnitude of Poisson's ratio with $z$ is present for all curves, which is consistent with the visual impression of the videos. It is not entirely clear why both methods give such different results but it is related to the very heterogeneous nature of the strain maps arising from the rather complex microstructure.

Fig. 19(b) shows the evolution of Poisson's ratios for load step 2. The same trends are present as for load step 1, except that the auxetic effect is much less pronounced. This is consistent with 
the fact that the unfolding power of the wrinkled microstructure decreases with the amount of tensile strain up to a point where Poisson's ratio tends towards zero, as reported in [49]. In that paper, tensile strains larger than 0.5 were necessary to see this locking effect whereas here, the total tensile strain is only about 0.025 but the compression ratios reported in that paper where much higher than here.

\section{Conclusion and future work}

The main conclusions of the current study are as follows.

- It has been possible to perform volume strain measurements on low density polymeric foams by coupling X-ray Computed Tomography and Digital Volume Correlation.

- Volume strain maps were thus obtained on conventional and standard foam specimens tested in tension over several load steps.

- The standard foam specimen exhibited reasonably homogeneous strain distributions, with positive Poisson's ratios. However, the increase of Poisson's ratio with longitudinal strain as well as unequal $\nu_{z x}$ and $\nu_{z y}$ values are still unexplained. Values above 0.5 at the last load step suggests some load induced stiffness anisotropy but this is yet to be confirmed.

- The auxetic specimen showed very heterogeneous strain distributions, probably because of its complex shriveled microstructure. Zones of positive and negative transverse strains clearly demonstrated that the auxetic behaviour is not uniform throughout the foam. This complex behaviour is to be connected with the results from [50] where random perturbations of a regular array of cells produced erratic auxetic and non-auxetic behaviour.

- All the evidence reported in this article suggests that the standard to auxetic conversion process was not uniform for the auxetic specimen. The field of view clearly exhibited two zones with different properties:

- Slices 1 to 17: lower axial modulus, lower magnitude of auxetic Poisson's ratio, higher strain heterogeneities.

- Slices 18 to 26: higher axial modulus, higher magnitude of auxetic Poisson's ratio, lower strain heterogeneities. 
Future work is necessary to better understand the mechanical behaviour of such foams, as well as the relationship between the manufacturing process and the resulting mechanical properties. One possible way forward would be to image the compression deformation of the standard foam when squeezed into the mould using X-ray CT, and then, after curing, image the tensile and compressive deformation of the resulting auxetic specimens. This procedure would help definign optimal process parameters to obtain auxetic foam with specific properties in a reproducible way. IN general, X-ray CT and DVC represent a perfect set of tools to investigate the mechanical behaviour of low density polymerci foams under complex stress conditions.

\section{References}

[1] F. Pierron. Identification of Poisson's ratios of standard and auxetic low density polymeric foams from full-field measurements. Journal of Strain Analysis for Engineering Design, $45(4): 233-253,2010$.

[2] P. Trench, E.G. Little, D. Tocher, P.O. O’Donnell, and V. Lawlor. The performance of threedimensional strain rosettes evaluated when embedded into a sphere. Strain, 45(2):149-159, 2009.

[3] C. O’Driscoll, E. O’Donnell, W. Stanley, P. O'Donnell, and E.G. Little. A comparison of three-dimensional embedded strain transducers, compression tested within the same strain field while offset at an angle with the principal axes. Strain, 48(5):375-387, 2012.

[4] R. Muthupillai, D. J. Lomas, P. J. Rossman, J. F. Greenleaf, A. Manduca, and R. L. Ehman. Magnetic resonance elastography by direct visualization of propagating acoustic strain waves. Science, 269(5232):1854-1857, 1995.

[5] A. Manduca, T.E. Oliphant, M.A. Dresner, J.L. Mahowald, S. A. Kruse, E. Amromin, J. P. Felmlee, J. F. Greenleaf, and R. L. Ehman. Magnetic resonance elastography: Non-invasive mapping of tissue elasticity. Medical Image Analysis, 5(4):237-254, 2001.

[6] R. Sinkus, M. Tanter, S. Catheline, J. Lorenzen, C. Kuhl, E. Sondermann, and M. Fink. Imaging anisotropic and viscous properties of breast tissue by magnetic resonanceelastography. Magnetic Resonance in Medicine, 53(2):372-387, 2005. 
[7] Bayly P.V., Black E.E, Pedersen R.C., Leister E.P., and Genin G.M. In vivo imaging of rapid deformation and strain in an animal model of traumatic brain injury. Journal of Biomechanics, 39:1086-1095, 2006.

[8] A. Pesavento, A. Lorenz, and H. Ermert. System for real-time elastography. Electronics Letters, 35(11):941-942, 1999.

[9] W. Khaled, S. Reichling, O.T. Bruhns, and H. Ermert. Ultrasonic strain imaging and reconstructive elastography for biological tissue. Ultrasonics, 44:199-202, 2006.

[10] M.H. De La Torre Ibarra, P.D. Ruiz, and J.M. Huntley. Simultaneous measurement of inplane and out-of-plane displacement fields in scattering media using phase-contrast spectral optical coherence tomography. Optics Letters, 34(6):806-808, 2009.

[11] P.D. Ruiz, J.M. Huntley, and A. Maranon. Tilt scanning interferometry: A novel technique for mapping structure and three-dimensional displacement fields within optically scattering media. Proceedings of the Royal Society A: Mathematical, Physical and Engineering Sciences, 462(2072):2481-2502, 2006.

[12] P.D. Ruiz, J.M. Huntley, and R.D. Wildman. Depth-resolved whole-field displacement measurement by wavelength-scanning electronic speckle pattern interferometry. Applied Optics, 44(19):3945-3953, 2005.

[13] P.D. Ruiz, J.M. Huntley, and J.M. Coupland. Depth-resolved imaging and displacement measurement techniques viewed as linear filtering operations. Experimental Mechanics, $51(4): 453-465,2011$.

[14] G. Restivo and G.L. Cloud. Embedded digital speckle pattern interferometry for threedimensional strain analysis. Experimental Mechanics, 48(6):731-740, 2008.

[15] R.A. Owen, R.V. Preston, P.J. Withers, H.R. Shercliff, and P.J. Webster. Neutron and synchrotron measurements of residual strain in an aluminium alloy TIG weld. Materials Science and Engineering A, 346:159-167, 2003. 
[16] B.K. Bay, T.S. Smith, D.P. Fyhrie, and M. Saad. Digital volume correlation: Threedimensional strain mapping using X-ray tomography. Experimental Mechanics, 39(3):217$226,1999$.

[17] E. Maire and P.J. Withers. Quantitative X-ray tomography. International Materials Reviews, 2013. In Press.

[18] Sutton M.A., Orteu J.-J., and Schreier H. Image correlation for shape, motion and deformation measurements. Springer, 2009.

[19] A. Germaneau, P. Doumalin, and J.C. Dupré. 3D strain field measurement by correlation of volume images using scattered light: Recording of images and choice of marks. Strain, $43(3): 207-218,2007$.

[20] A. Germaneau, P. Doumalin, and J.C. Dupré. Full 3D measurement of strain field by scattered light for analysis of structures. Experimental Mechanics, 47(4):523-532, 2008.

[21] A. Germaneau, P. Doumalin, and J.C. Dupré. Comparison between X-ray micro-computed tomography and optical scanning tomography for full 3D strain measurement by digital volume correlation. NDT and E International, 41(6):407-415, 2008.

[22] J. Fu, M. Haghighi-Abayneh, F. Pierron, and P.D. Ruiz. Assessment of corneal deformation using optical coherence tomography and digital volume correlation. In Society for Experimental Mechanics, editor, Proceedings of the SEM Annual Conference June 11-14 in Costa Mesa, CA, USA, 2012.

[23] C. Franck, S. Hong, S.A. Maskarinec, D.A. Tirrell, and G. Ravichandran. Three-dimensional full-field measurements of large deformations in soft materials using confocal microscopy and digital volume correlation. Experimental Mechanics, 47(3):427-438, 2008.

[24] A. Benoit, S. Guérard, B. Gillet, G. Guillot, F. Hild, D. Mitton, J.-N. Périé, and S. Roux. 3D analysis from micro-MRI during in situ compression on cancellous bone. Journal of Biomechanics, 42(14):2381-2386, 2009.

[25] A. Kak and M. Slaney. Principles of Computerized Tomographic Imaging. IEEE Press, New York, 1988. 
[26] E.N. Landis and D.T. Keane. X-ray microtomography. Materials Characterization, 61(12):1305-1316, 2010.

[27] H. Toda, I. Sinclair, J.-Y. Buffière, E. Maire, K.H. Khor, P. Gregson, and T. Kobayashi. A 3D measurement procedure for internal local crack driving forces via synchrotron X-ray microtomography. Acta Materialia, 52(5):1305-1317, 2004.

[28] A.J. Moffat, P. Wright, J.-Y. Buffière, I. Sinclair, and S.M. Spearing. Micromechanisms of damage in $0^{\circ}$ splits in a [90/0]s composite material using synchrotron radiation computed tomography. Scripta Materialia, 59(10):1043-1046, 2008.

[29] C. Chateau, L. Gélébart, M. Bornert, J. Crépin, E. Boller, C. Sauder, and W. Ludwig. In situ X-ray microtomography characterization of damage in $\mathrm{SiCf} / \mathrm{SiC}$ minicomposites. Composites Science and Technology, 71(6):916-924, 2011.

[30] F. Forsberg and C. Siviour. 3D deformation and strain analysis in compacted sugar using Xray microtomography and digital volume correlation. Measurement Science and Technology, 20(9):art. no. 095703, 2009.

[31] S.A. Hall, N. Lenoir, and G. Viggiani. Full-field characterisation of strain localisation evolution in sand under triaxial loading by in-situ X-ray microtomography and 3D-volumetric digital image correlation. In Society of Petroleum Engineers, editor, 71st European Association of Geoscientists and Engineers Conference and Exhibition 2009, volume 4, pages 2327-2331, 2009.

[32] H. Proudhon, A. Moffat, I. Sinclair, and J.-Y. Buffière. Three-dimensional characterisation and modelling of small fatigue corner cracks in high strength Al-alloys. Comptes Rendus Physique, 13(3):316-327, 2012.

[33] S. Roux, F. Hild, P. Viot, and D. Bernard. Three-dimensional image correlation from X-ray computed tomography of solid foam. Composites Part A, 39(8):1253-1265, 2008.

[34] S.A. McDonald, N. Ravirala, P.J. Withers, and A. Alderson. In situ three-dimensional X-ray microtomography of an auxetic foam under tension. Scripta Materialia, 60:232-235, 2009. 
[35] F. Forsberg, M. Sjödahl, R. Mooser, E. Hack, and P. Wyss. Full three-dimensional strain measurements on wood exposed to three-point bending: Analysis by use of digital volume correlation applied to synchrotron radiation micro-computed tomography image data. Experimental Mechanics, 46(1):47-60, 2010.

[36] B.K. Bay. Experimental measurement of three-dimensional continuum-level strain fields in trabecular bone. Advances in Experimental Medicine and Biology, 496(3):181-197, 2001.

[37] T.S. Smith, B.K. Bay, and M.M. Rashid. Digital volume correlation including rotational degrees of freedom during minimization. Experimental Mechanics, 42(3):272-278, 2002.

[38] M. Gates, J. Lambros, and M.T. Heath. Towards high performance digital volume correlation. Experimental Mechanics, 51(4):491-507, 2011.

[39] I. Jandejsek, O. Jiroušek, and D. Vavrìk. Precise strain measurement in complex materials using digital volumetric correlation and time lapse micro-CT data. Procedia Engineering, 10:1730-1735, 2011.

[40] N. Limodin, J. Réthoré, J.-Y. Buffière, A. Gravouil, F. Hild, and S. Roux. Crack closure and stress intensity factor measurements in nodular graphite cast iron using three-dimensional correlation of laboratory X-ray microtomography images. Acta Materialia, 57(14):40904101, 2009.

[41] N. Limodin, J. Réthoré, J.-Y. Buffière, F. Hild, S. Roux, W. Ludwig, J. Rannou, and A. Gravouil. Influence of closure on the 3D propagation of fatigue cracks in a nodular cast iron investigated by X-ray tomography and 3D volume correlation. Acta Materialia, $58(8): 2957-2967,2010$.

[42] N. Lenoir, M. Bornert, J. Desrues, P. Bésuelle, and C. Viggiani. Volumetric digital image correlation applied to X-ray microtomography images from triaxial compression tests on argillaceous rock. Strain, 43(3):193-205, 2007.

[43] www.lavision.de. Davis digital volume correlation (DVC) package.

[44] F.-P. Chiang, G. Uzer, and J. Yu. Auxetic PVC foam as a new core material for sandwich structures. In American Society for Composites, editor, 24th Annual Technical Conference 
of the American Society for Composites 2009 and 1st Joint Canadian-American Technical Conference on Composites, volume 3, pages 1667-1677, 2009.

[45] A. Alderson, J. Rasburn, and K.E. Evans. Mass transport properties of auxetic (negative poisson's ratio) foams. Physica Status Solidi (b), 244(3):817-827, 2007.

[46] A. Alderson and K.L. Evans. Auxetic materials. Proceedings of the IMechE, Part G: Journal of Aerospace Engineering, 221:565-575, 2007.

[47] F. Scarpa A. Bezazi. Mechanical behaviour of conventional and negative Poisson's ratio thermoplastic polyurethane foams under compressive cyclic loading. International Journal of Fatigue, 29:922-930, 2007.

[48] S.A. McDonald, G. Dedreuil-Monet, Y.T. Yao, A. Alderson, and P.J. Withers. In situ 3D X-ray microtomography study comparing auxetic and non-auxetic polymeric foams under tension. Physica Status Solidi (B), 248(1):45-51, 2011.

[49] F. Scarpa, P. Pastorino, A. Garelli, S. Patsias, and M. Ruzzene. Auxetic compliant flexible PU foams static and dynamic properties. Physica Status Solidi B, 242(3):681-694, 2005.

[50] E.J. Horrigan, C.W. Smith, F.L. Scarpa, N. Gaspar, A.A. Javadi, M.A. Berger, and K.E. Evans. Simulated optimisation of disordered structures with negative Poisson's ratios. Mechanics of Materials, 41(8):919-927, 2009. 


\section{Supporting Information}

This section contains some videos associated with the tests described in the paper. The videos

can be viewed at http://www.camfit.fr/Supp_Pierron_Foams_2012.php

Video 1: First $100(\mathrm{x}, \mathrm{y})$ slices for the standard foam

Video 2: First $100(\mathrm{x}, \mathrm{y})$ slices for the auxetic foam

Video 3: $\varepsilon_{z z}$ for load step 1, standard foam 
Video 4: $\varepsilon_{x x}$ for load step 1, standard foam

Video 5: $\varepsilon_{y y}$ for load step 1, standard foam

Video 6: $\varepsilon_{x y}$ for load step 1, standard foam

Video 7: $\varepsilon_{x z}$ for load step 1, standard foam

Video 8: $\varepsilon_{y z}$ for load step 1, standard foam

Video 9: $\varepsilon_{z z}$ for load step 1, auxetic foam

Video 10: $\varepsilon_{x x}$ for load step 1, auxetic foam

Video 11: $\varepsilon_{y y}$ for load step 1, auxetic foam

Video 12: $\varepsilon_{x y}$ for load step 1, auxetic foam

Video 13: $\varepsilon_{x z}$ for load step 1, auxetic foam

Video 14: $\varepsilon_{y z}$ for load step 1, auxetic foam

Video 15: $\nu_{z x}$ for load step 1, auxetic foam

Video 16: $\nu_{z y}$ for load step 1, auxetic foam 


\section{List of Tables}

1 Experimental details concerning the four sets of CT experiments . . . . . . . 24

2 Details concerning Digital Volume Correlation . . . . . . . . . . . . . . 25 
Table 1: Experimental details concerning the four sets of CT experiments

\begin{tabular}{lcccc}
\hline Volume names & $\begin{array}{c}\text { Volume size } \\
\left(\operatorname{voxel}^{3}\right)\end{array}$ & $\begin{array}{c}\text { Voxel size } \\
\left(\mu \mathrm{m}^{3}\right)\end{array}$ & $\begin{array}{c}\text { Size of field of view } \\
\left(\mathrm{mm}^{3}\right)\end{array}$ & Load steps \\
\hline $\begin{array}{c}\text { Auxetic unloaded } \\
\text { SA1, SA2 and TA }\end{array}$ & $800 \times 800 \times 679$ & $15 \times 15 \times 15$ & $12 \times 12 \times 10.2$ & 2 (station. and transl.) \\
$\begin{array}{c}\text { Auxetic tension } \\
\text { AA, AB and AC }\end{array}$ & $700 \times 700 \times 676$ & $15 \times 15 \times 15$ & $10.5 \times 10.5 \times 10.1$ & 2 \\
$\begin{array}{l}\text { Standard unloaded } \\
\quad \text { SS1, SS2 and TS }\end{array}$ & $1000 \times 1000 \times 1000$ & $15 \times 15 \times 15$ & $15 \times 15 \times 15$ & 2 (station. and transl.) \\
$\begin{array}{l}\text { Standard tension } \\
\quad \text { SA, SB, SC and SD }\end{array}$ & $633 \times 633 \times 558$ & $15 \times 15 \times 15$ & $9.5 \times 9.5 \times 8.4$ & 3 \\
\hline
\end{tabular}


Table 2: Details concerning Digital Volume Correlation

\begin{tabular}{lccc}
\hline Volume names & $\begin{array}{c}\text { Subset size } \\
\left(\text { voxel }^{3}\right)\end{array}$ & $\begin{array}{c}\text { Shift } \\
(\%)\end{array}$ & $\begin{array}{c}\text { Size of displacement } \\
\text { data set }\end{array}$ \\
\hline $\begin{array}{c}\text { Auxetic unloaded } \\
\text { SA1, SA2 and TA }\end{array}$ & $48 \times 48 \times 48$ & 50 & $33 \times 33 \times 28$ \\
$\begin{array}{c}\text { Auxetic tension } \\
\text { AA, AB and AC }\end{array}$ & $48 \times 48 \times 48$ & 50 & $29 \times 29 \times 28$ \\
$\begin{array}{c}\text { Standard unloaded } \\
\text { SS1, SS2 and TS }\end{array}$ & $64 \times 64 \times 64$ & 50 & $31 \times 31 \times 31$ \\
$\begin{array}{l}\text { Standard tension } \\
\text { SA, SB, SC and SD }\end{array}$ & $64 \times 64 \times 64$ & 50 & $20 \times 20 \times 17$ \\
\hline
\end{tabular}




\section{List of Figures}

1 Experimental details: fixture, field of view and imaging parameters for the tensile test on the auxetic specimen . . . . . . . . . . . . . . 28

$22 \mathrm{D}$ views of $\mathrm{CT}$ images of both standard and auxetic foams, with same magnification. 29

3 Magnified views of auxetic and standard foams f . . . . . . . . . . . 30

$42 \mathrm{D}$ views of typical raw images for standard and auxetic foams, same magnification. Red squares indicate subset sizes selected for the paper, 64 for the standard foam and 48 for the auxetic one. . . . . . . . . . . . . . 31

5 Evolution of the standard deviation of the strain noise maps obtained with DVC from stationary auxetic and standard foam specimen. . . . . . . . . . . . . 32

6 Strain maps for slice 15, stationary specimen, auxetic foam. . . . . . . . . . . 33

7 Evolution of the standard deviation of the strain noise maps obtained with DVC from translated auxetic and standard foam specimen. . . . . . . . . . . . . . . 34

8 Evolution of the average $U_{z}$ displacement as a function of $\mathrm{z}$, for all load steps, standard specimen in tension. . . . . . . . . . . . . . . . . 35

9 Evolution of the average $\varepsilon_{z z}$ strain as a function of $z$, for all load steps, standard specimen in tension. Incremental strains between two consecutive load steps are represented here, not total strains. . . . . . . . . . . . . . 36

10 Six strain maps for slice 5 , standard foam in tension, load step 1 . . . . . . . . . 37

11 Evolution of the average $U_{z}$ displacement as a function of $\mathrm{z}$, for both load steps, auxetic specimen in tension. . . . . . . . . . . . . . . . . 38

12 Six strain maps for slice 13 , auxetic foam in tension, load step $1 . \quad \ldots . . . . . .39$

$13 \varepsilon_{y y}$ strain maps for slice 13 for both load steps, together with a typical noise map. Auxetic foam in tension. . . . . . . . . . . . . . . . . . . 40

14 Evolution of the standard deviation of the different strain components as a function of the $z$ position. Auxetic foam in tension. . . . . . . . . . . . . 41

15 Evolution of both Poisson's ratios calculated with the two methods of Section 6.1, for all three load steps. Standard foam in tension. . . . . . . . . . . . . . . 
16 Evolution of the average transverse strains $\varepsilon_{x x}$ and $\varepsilon_{y y}$ as a function of $z$, for the three load steps, standard foam in tension. . . . . . . . . . . . . . . . 43

17 Normal strain maps for slice 5, standard foam in tension, load step 2 . . . . . . . 44

18 Poisson's ratios and associated transverse strain maps, slice 13, load step 1, auxetic foam in tension. . . . . . . . . . . . . . . . . . . 45

19 Evolution of both Poisson's ratios calculated with the two methods of Section 6.1, for both load steps. Auxetic foam in tension. . . . . . . . . . . . . . . . . . 46 


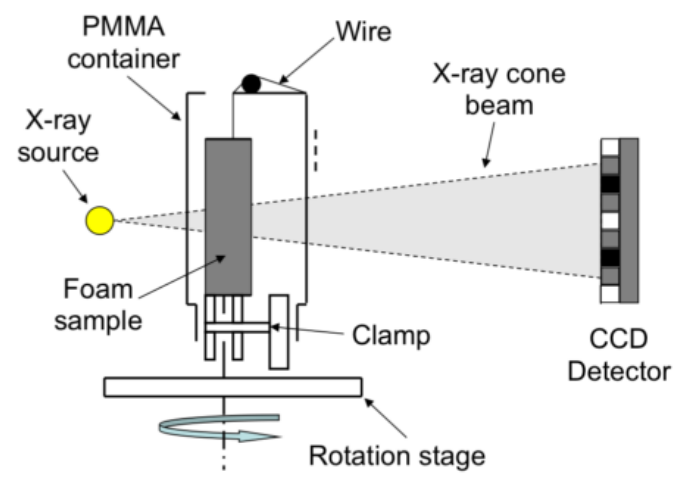

(A) Tensile test fixture

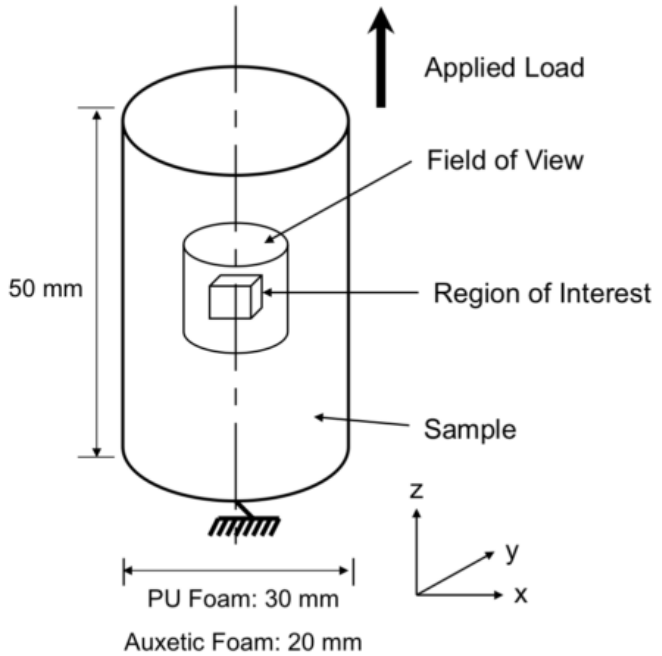

(B) Definition of sample dimensions

Figure 1: Experimental details: fixture, field of view and imaging parameters for the tensile test on the auxetic specimen 


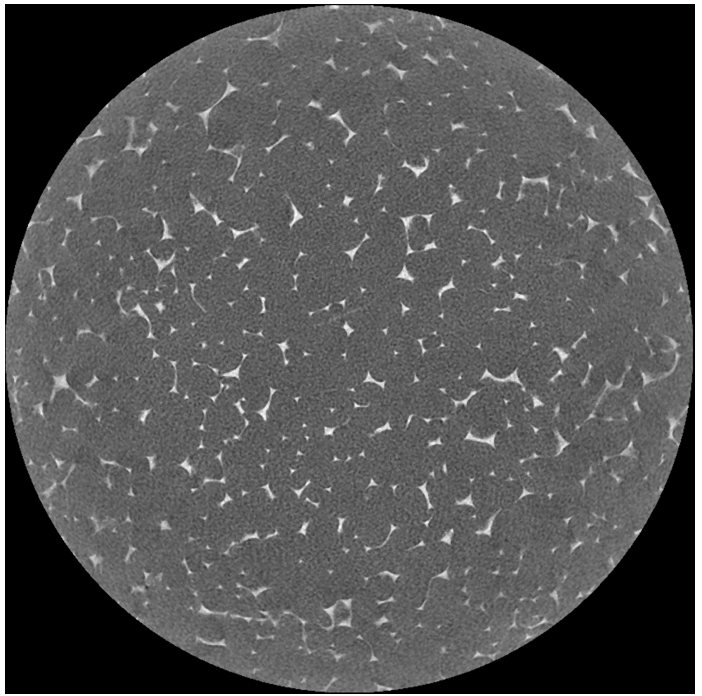

(A) Auxetic

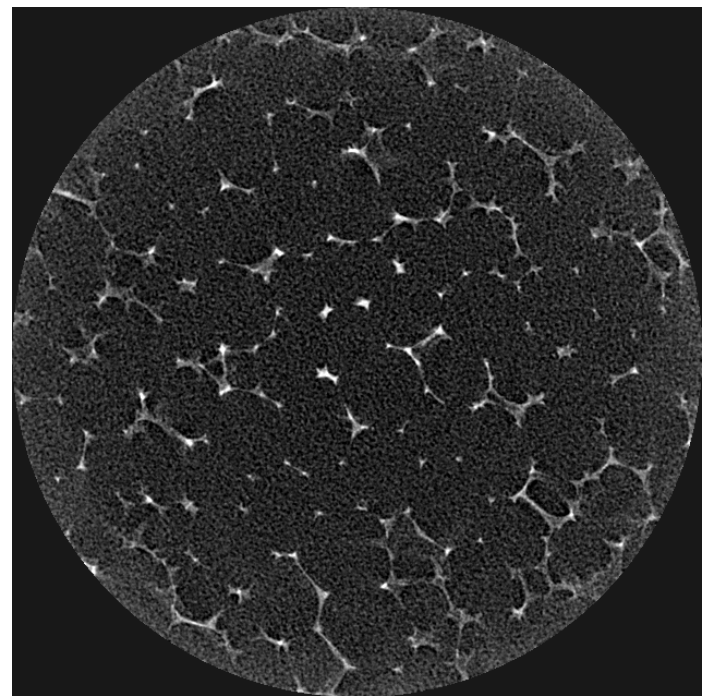

(в) Standard

Figure 2: 2D views of CT images of both standard and auxetic foams, with same magnification. 


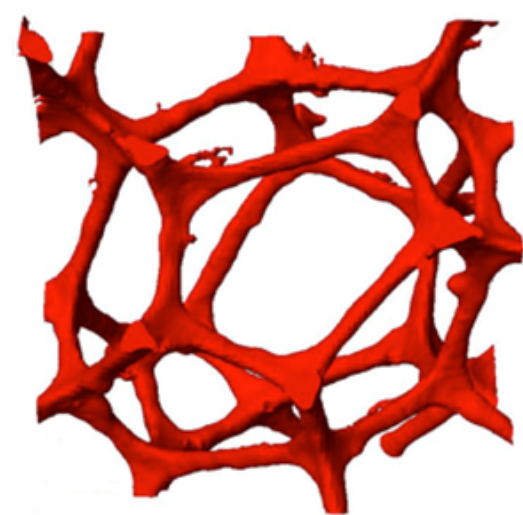

Conventional Foam

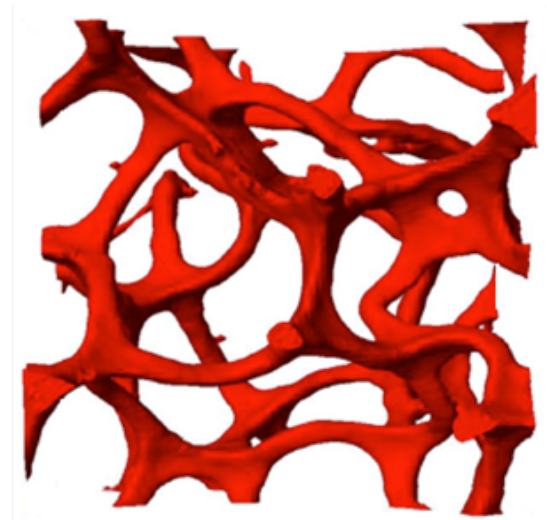

Auxetic Foam

(A) Rendered view of standard and auxetic foams

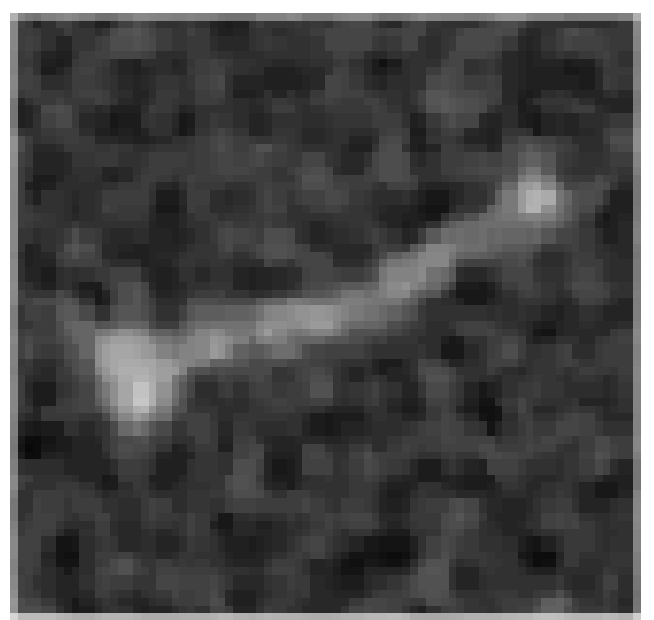

(B) Close-up view of cell wall, cell wall thickness around 6 voxels, ie, $0.1 \mathrm{~mm}$.

Figure 3: Magnified views of auxetic and standard foams 


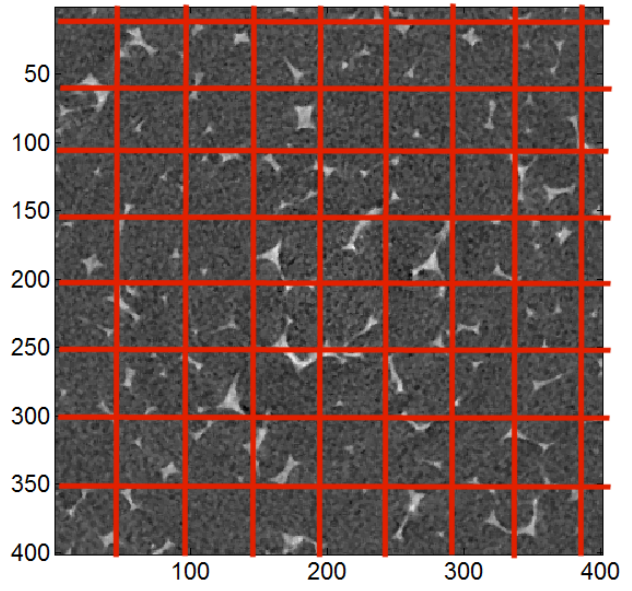

(A) Auxetic foam, 48 subset size

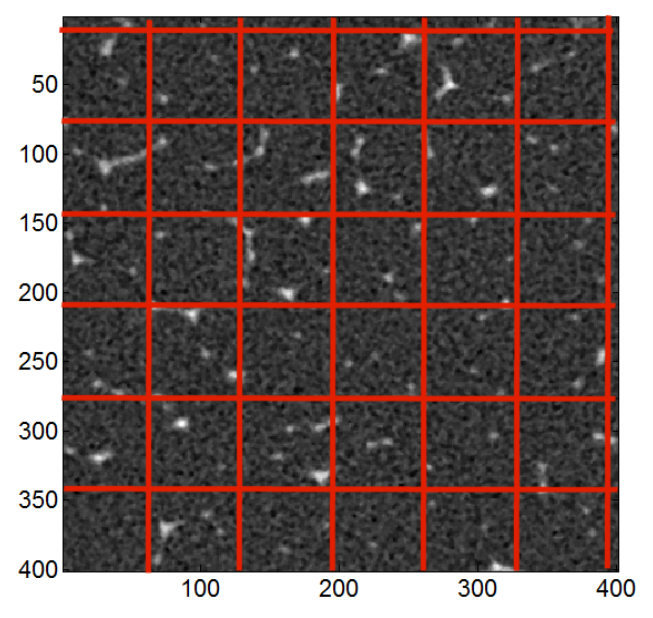

(в) Standard foam, 64 subset size

Figure 4: 2D views of typical raw images for standard and auxetic foams, same magnification. Red squares indicate subset sizes selected for the paper, 64 for the standard foam and 48 for the auxetic one. 


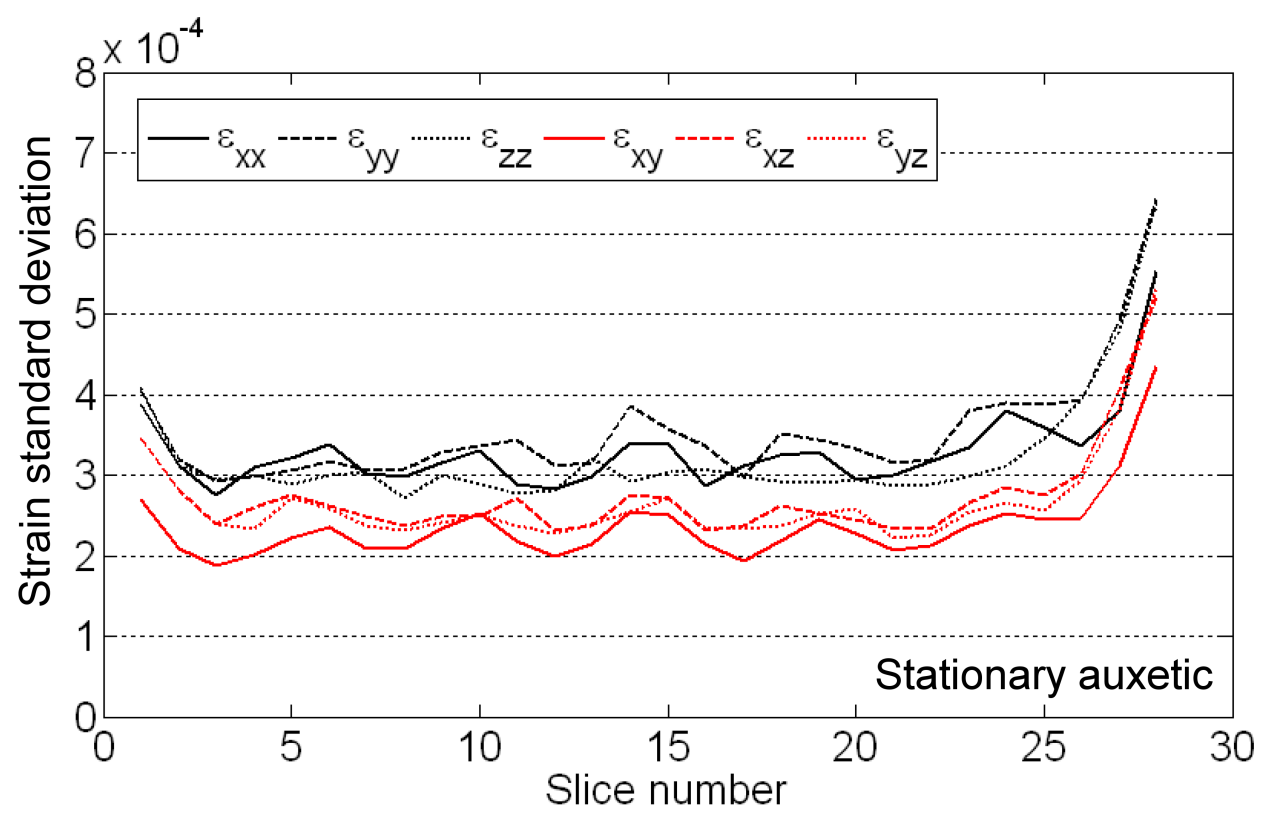

(A) Auxetic, stationary

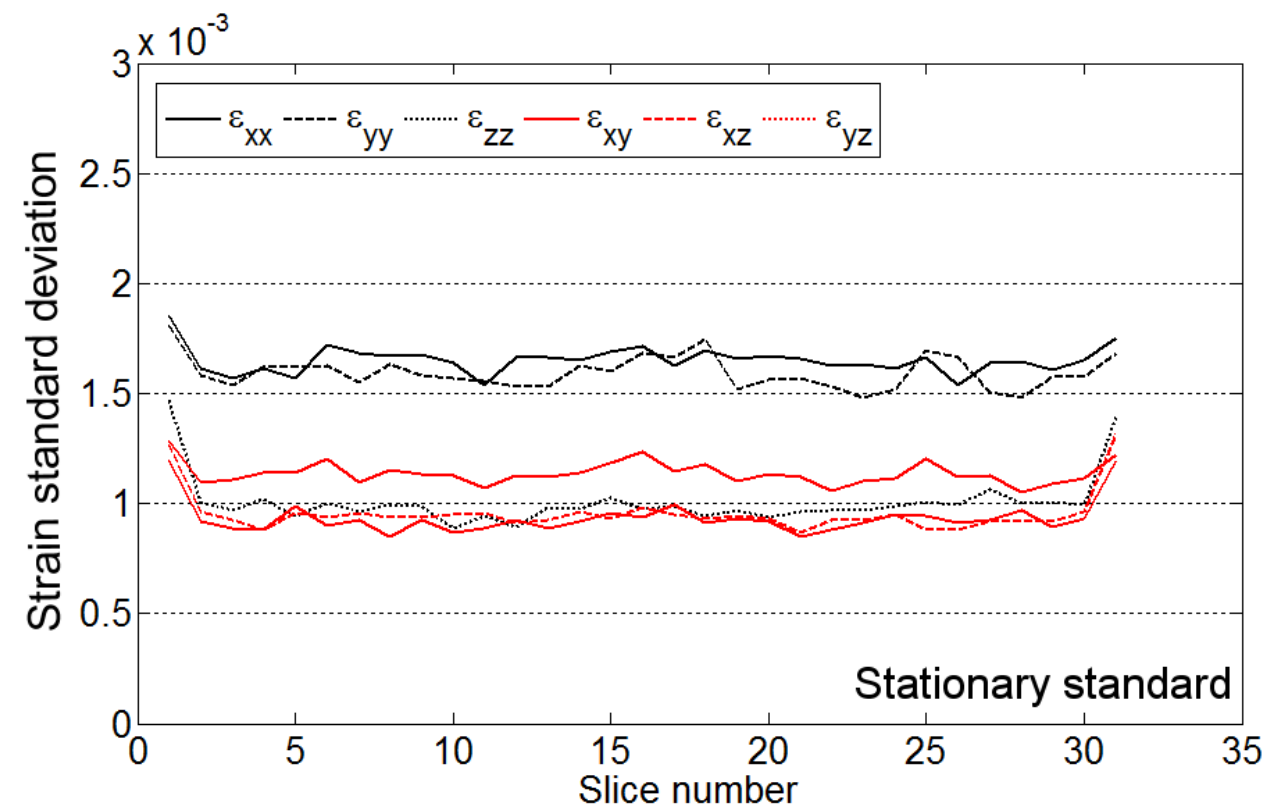

(B) Standard, stationary

Figure 5: Evolution of the standard deviation of the strain noise maps obtained with DVC from stationary auxetic and standard foam specimen. 

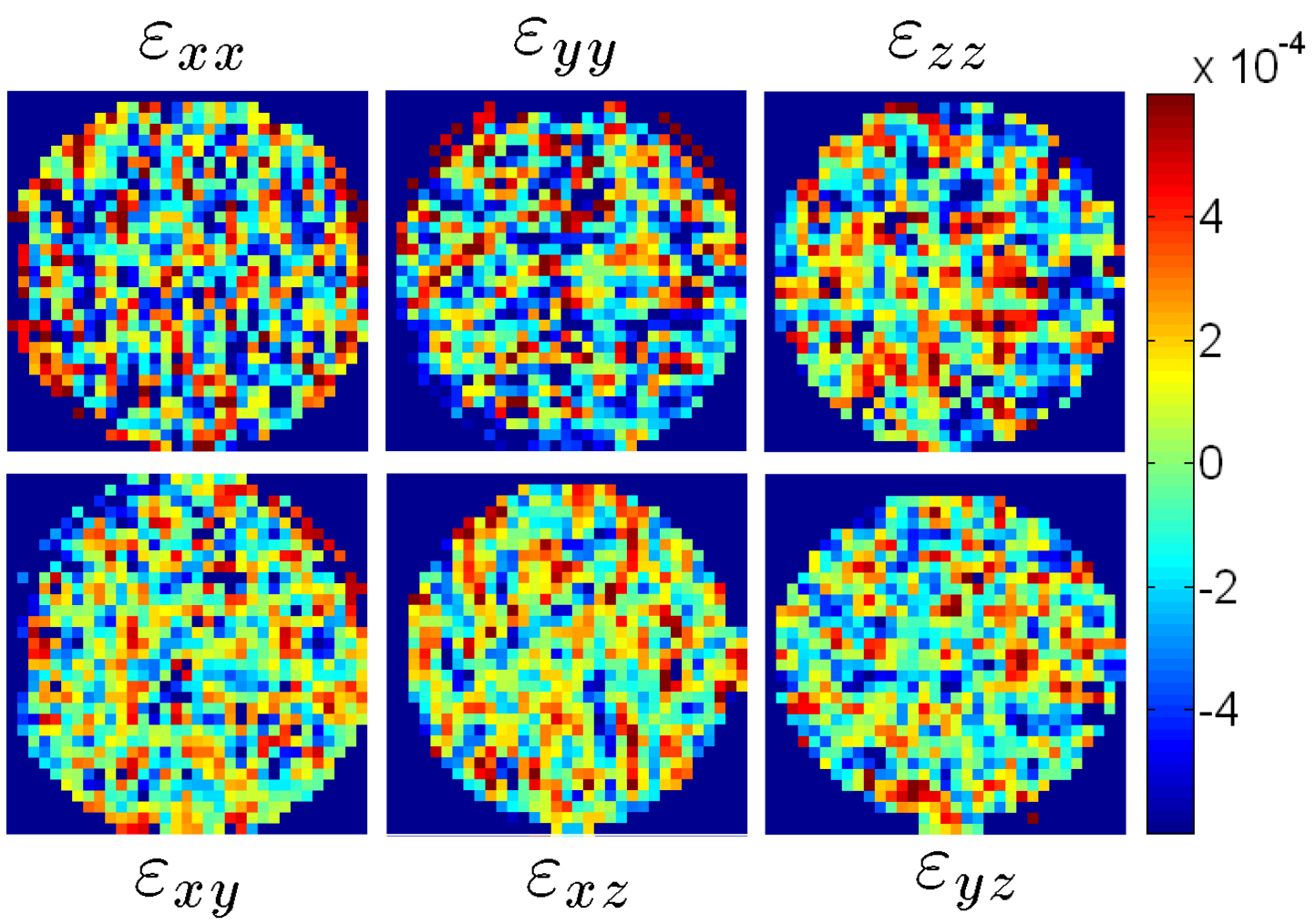

Figure 6: Strain maps for slice 15, stationary specimen, auxetic foam. 


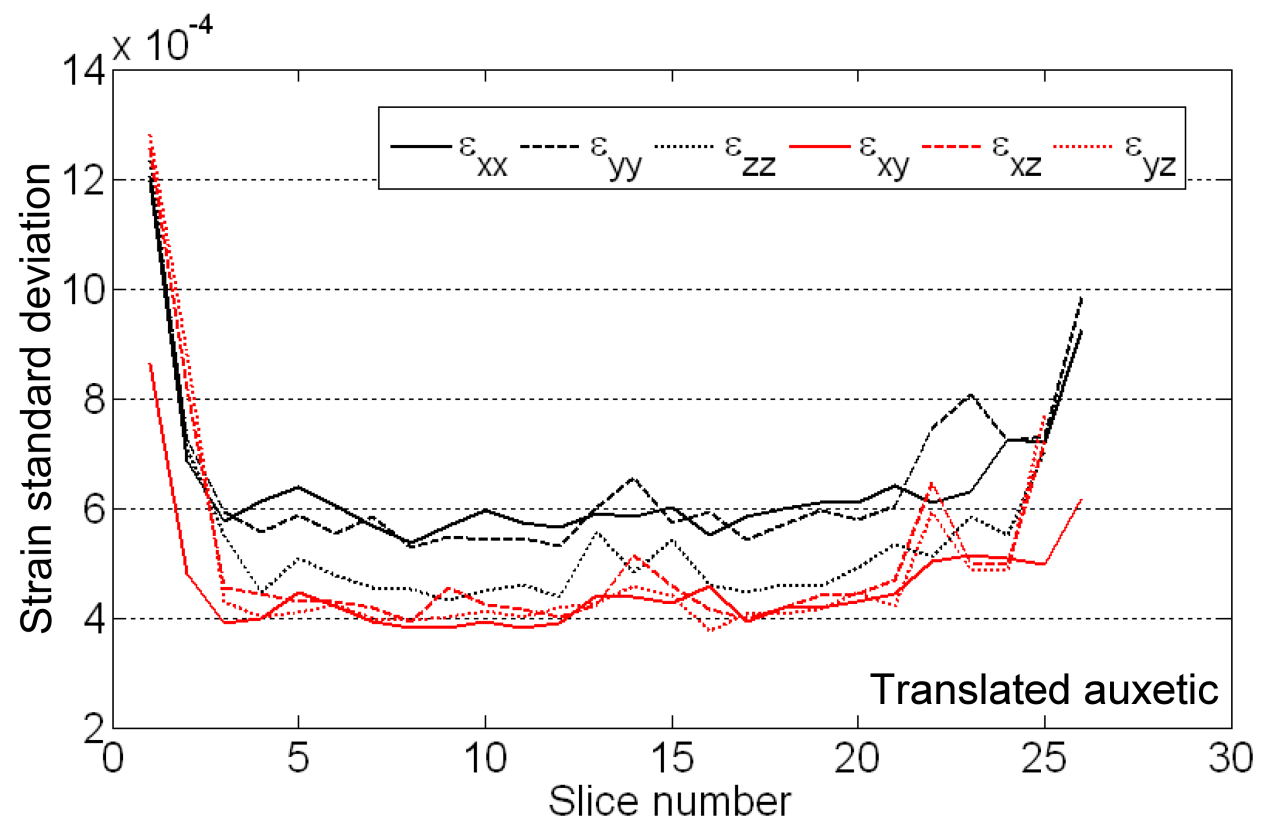

(A) Auxetic, translated

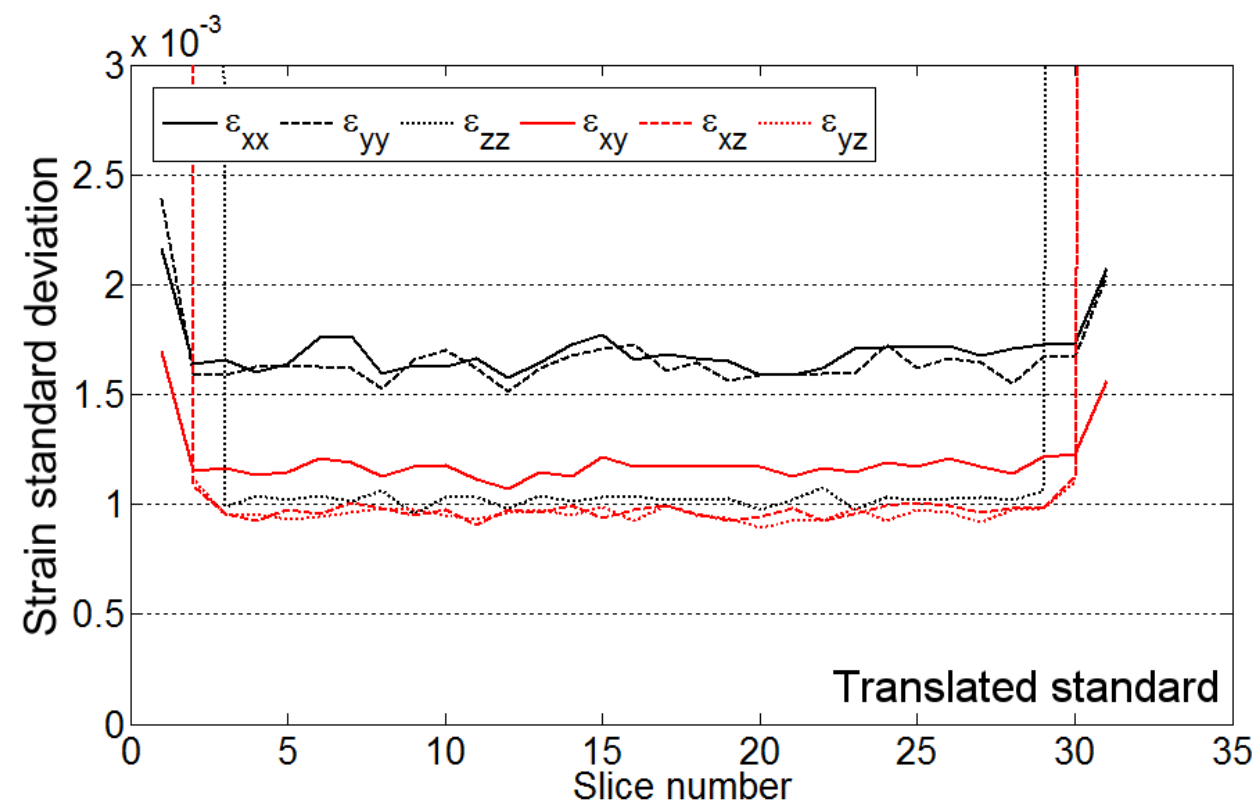

(B) Standard, translated

Figure 7: Evolution of the standard deviation of the strain noise maps obtained with DVC from translated auxetic and standard foam specimen. 


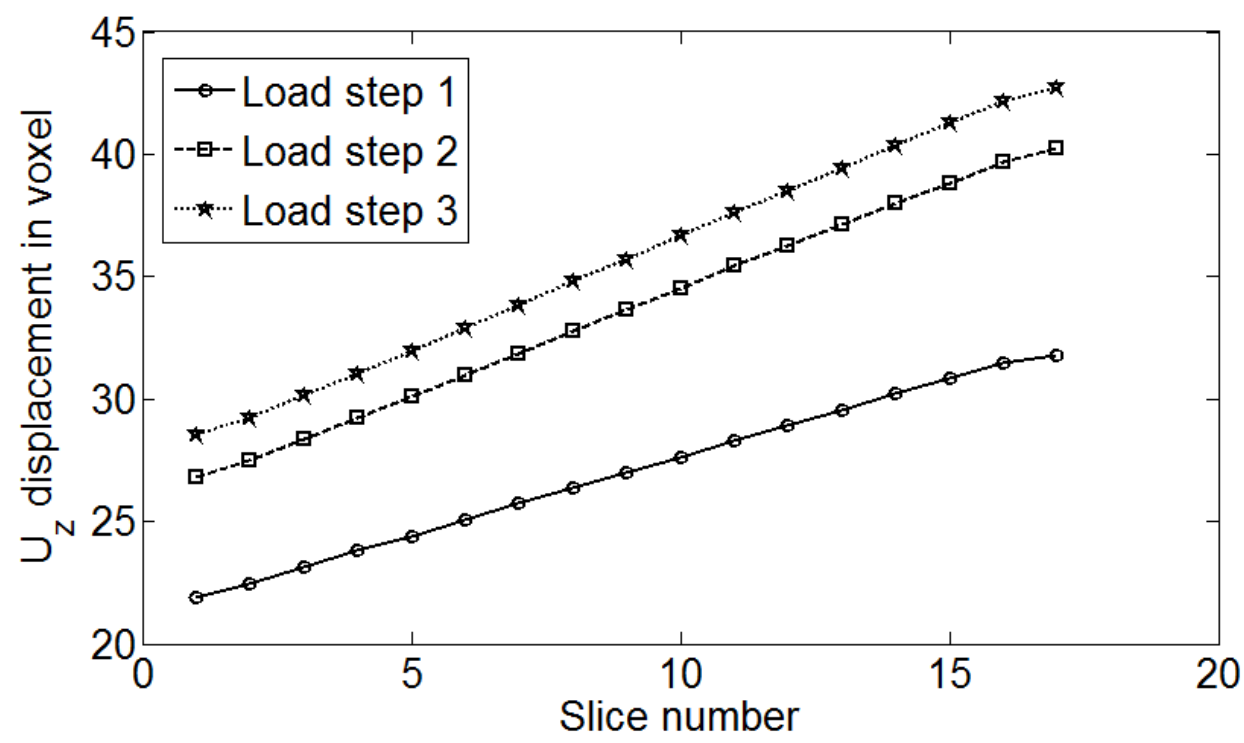

Figure 8: Evolution of the average $U_{z}$ displacement as a function of $\mathrm{z}$, for all load steps, standard specimen in tension. 


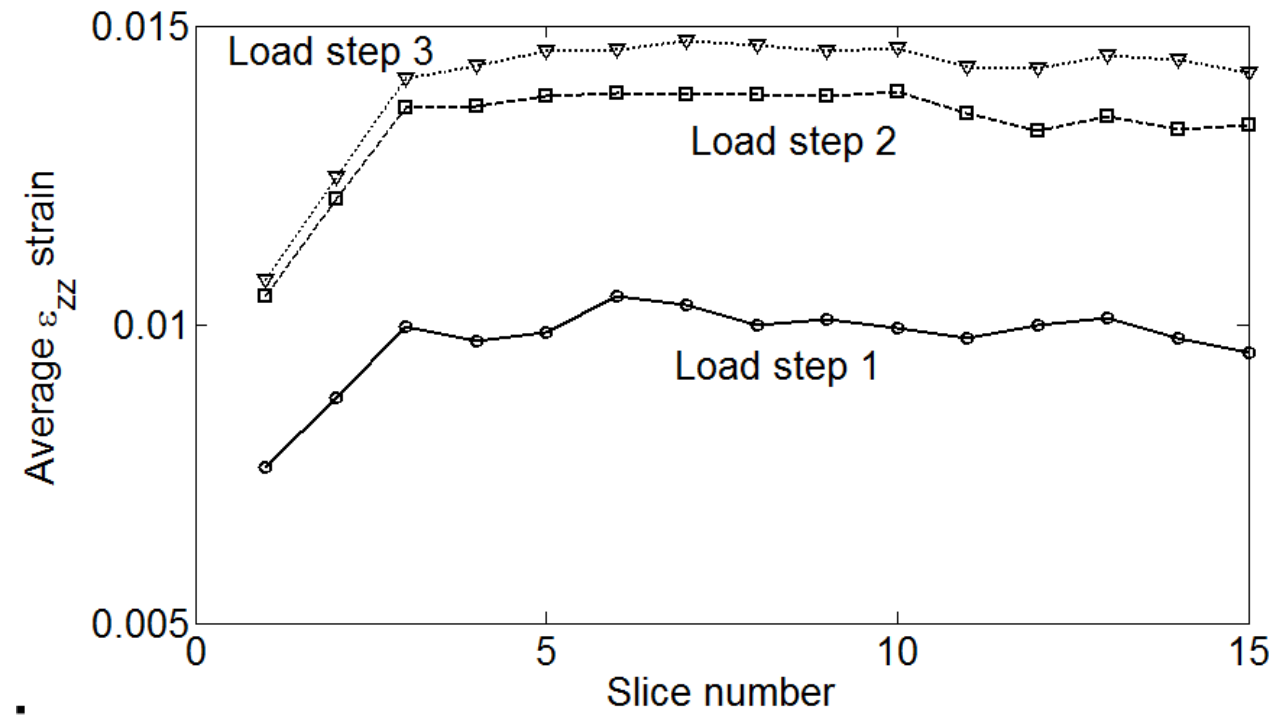

Figure 9: Evolution of the average $\varepsilon_{z z}$ strain as a function of $z$, for all load steps, standard specimen in tension. Incremental strains between two consecutive load steps are represented here, not total strains. 


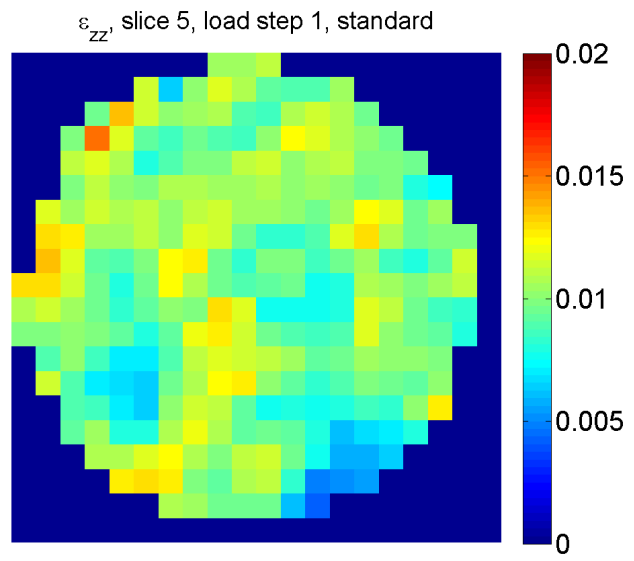

(A) $\varepsilon_{z z}$

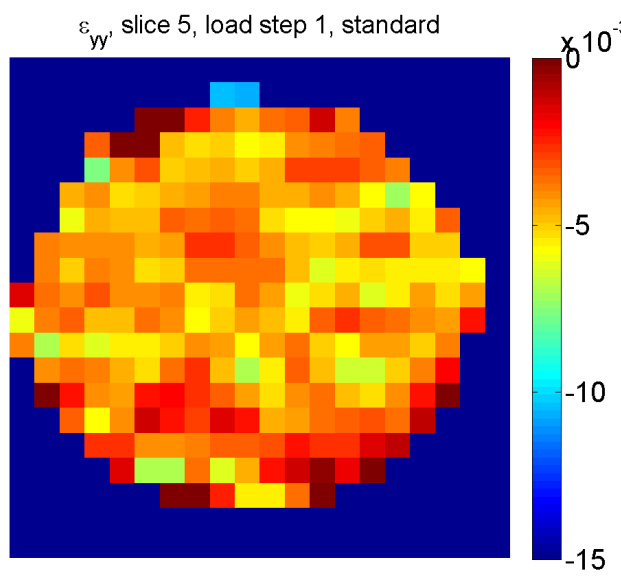

(C) $\varepsilon_{y y}$

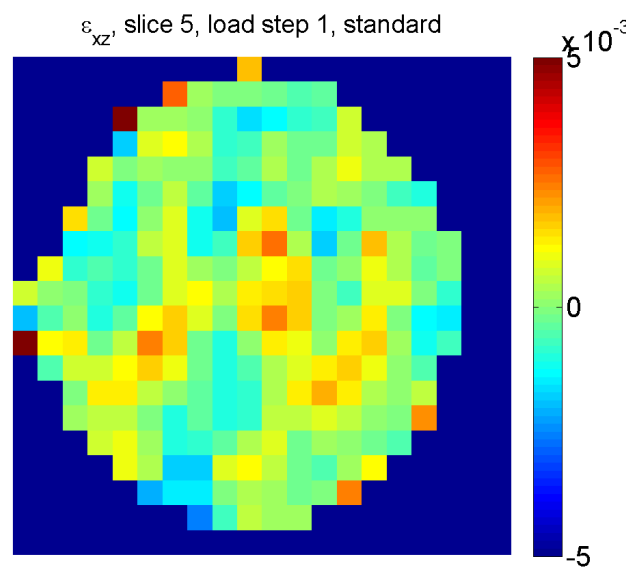

(E) $\varepsilon_{x z}$

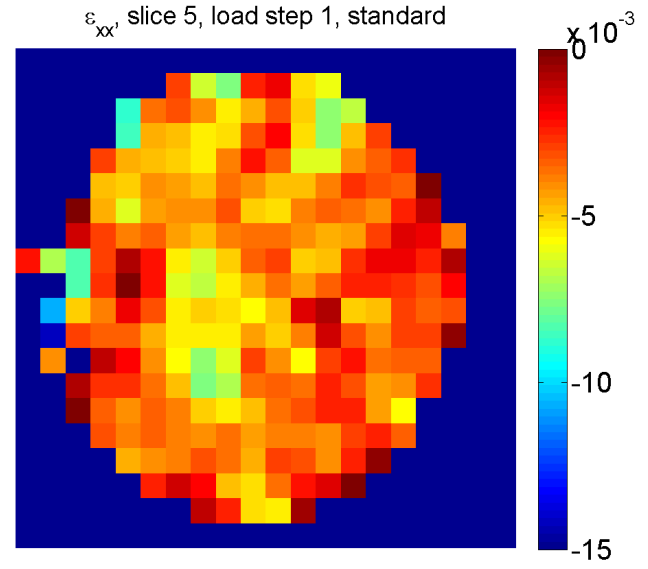

(B) $\varepsilon_{x x}$

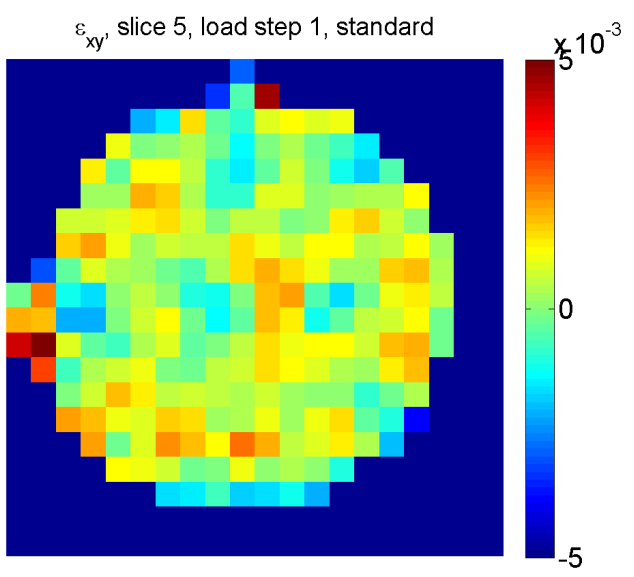

(D) $\varepsilon_{x y}$

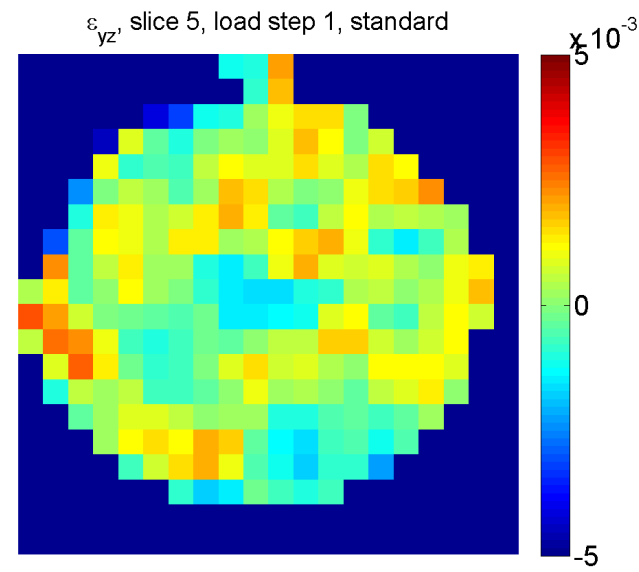

(F) $\varepsilon_{y z}$

Figure 10: Six strain maps for slice 5, standard foam in tension, load step 1. 


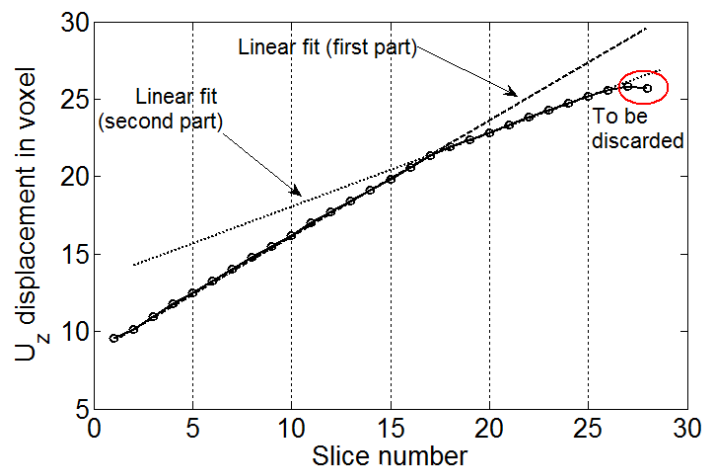

(A) Load step 1

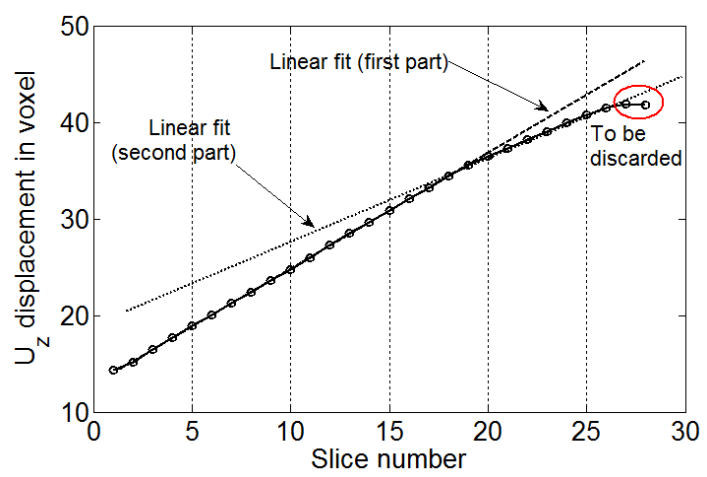

(B) Load step 2

Figure 11: Evolution of the average $U_{z}$ displacement as a function of $\mathrm{z}$, for both load steps, auxetic specimen in tension. 


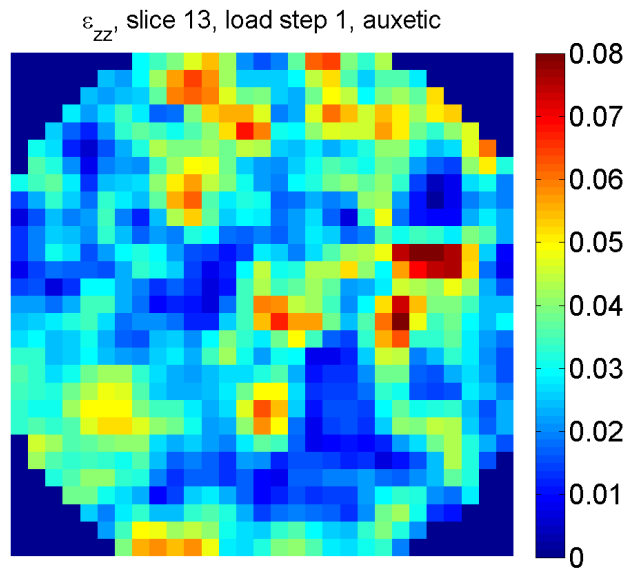

(A) $\varepsilon_{z z}$

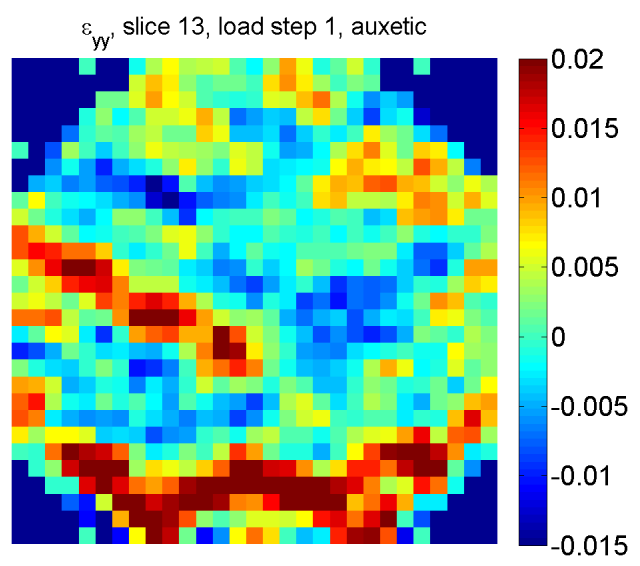

(C) $\varepsilon_{y y}$

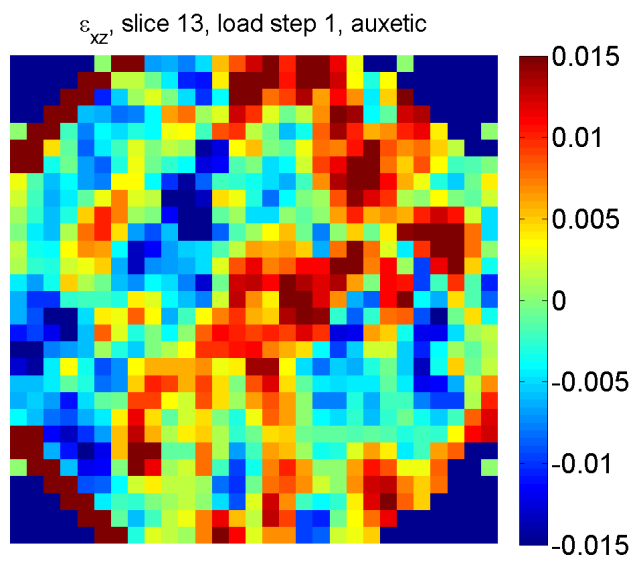

(E) $\varepsilon_{x z}$

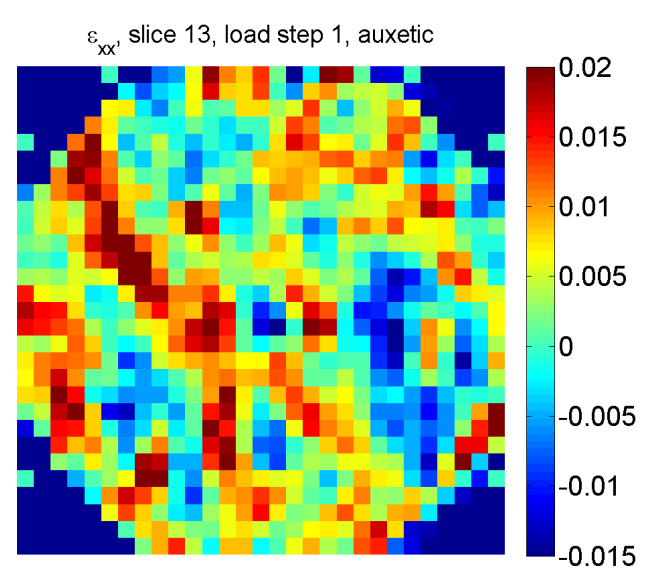

(B) $\varepsilon_{x x}$

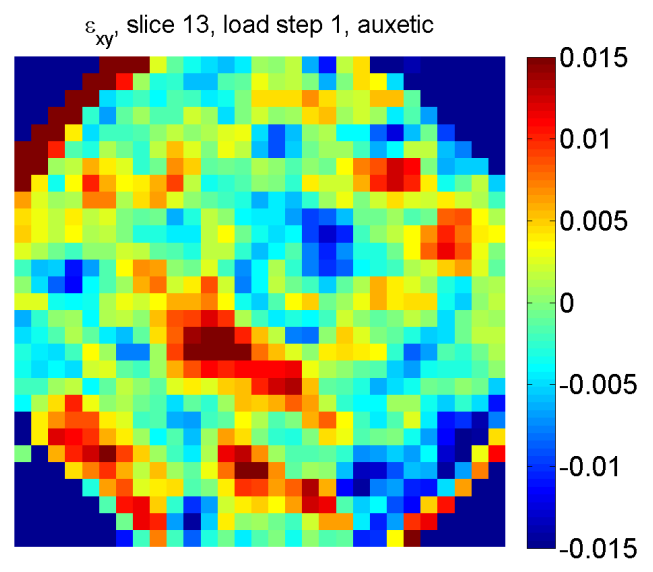

(D) $\varepsilon_{x y}$

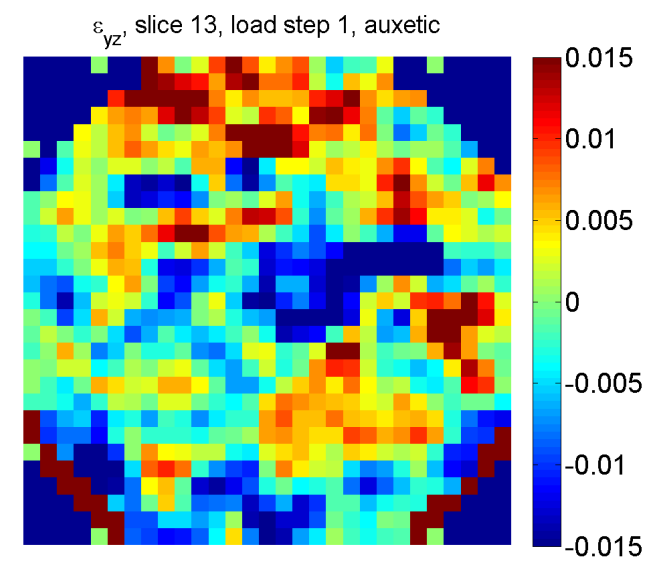

(F) $\varepsilon_{y z}$

Figure 12: Six strain maps for slice 13, auxetic foam in tension, load step 1. 


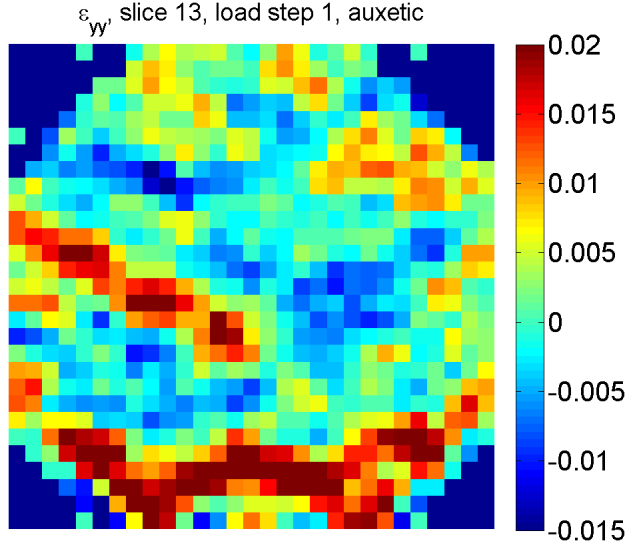

(A) $\varepsilon_{y y}$, slice 13 , load step 1

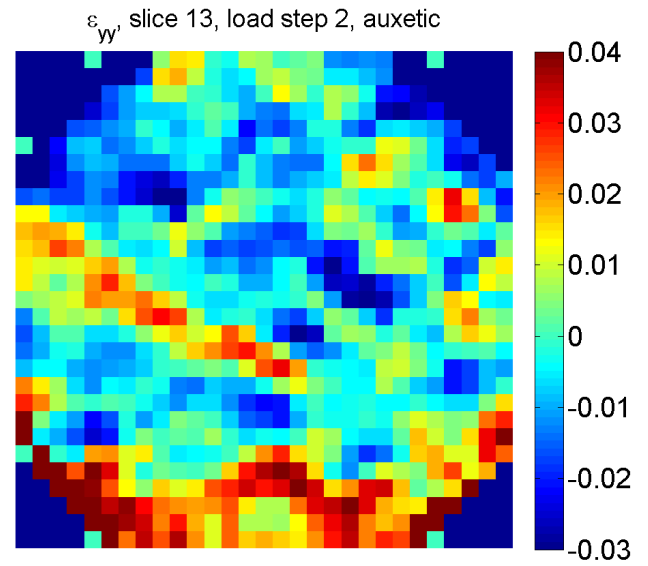

(в) $\varepsilon_{y y}$, slice 13 , load step 2

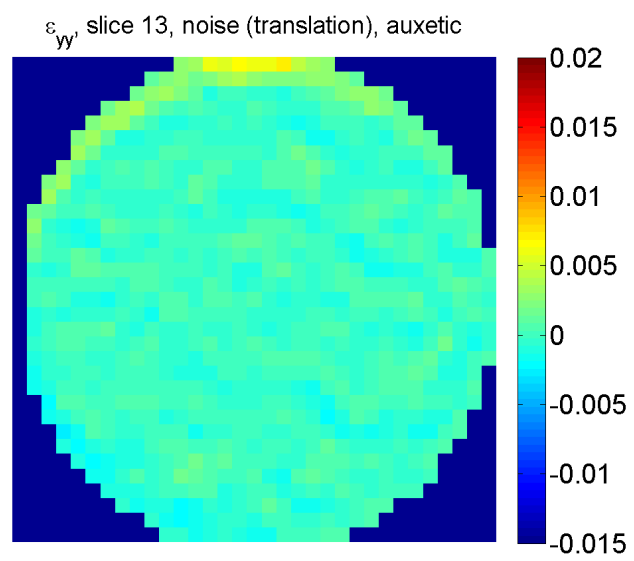

(C) $\varepsilon_{y y}$, noise map

Figure 13: $\varepsilon_{y y}$ strain maps for slice 13 for both load steps, together with a typical noise map. Auxetic foam in tension. 


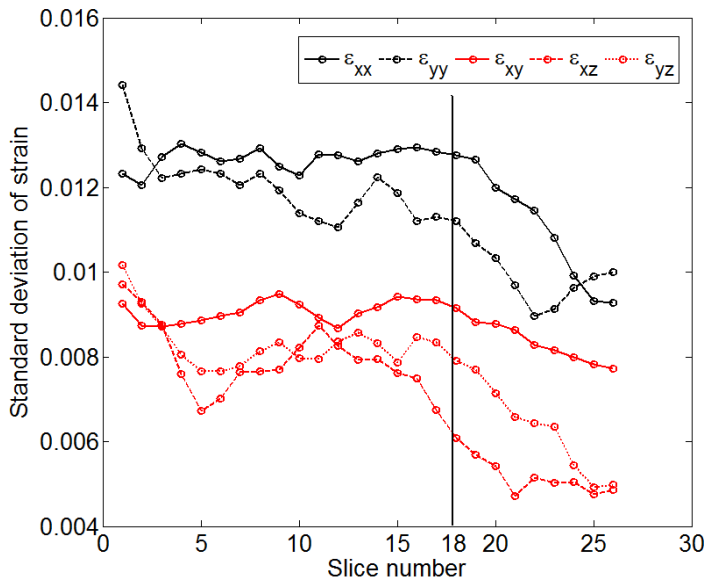

(A) Load step 1

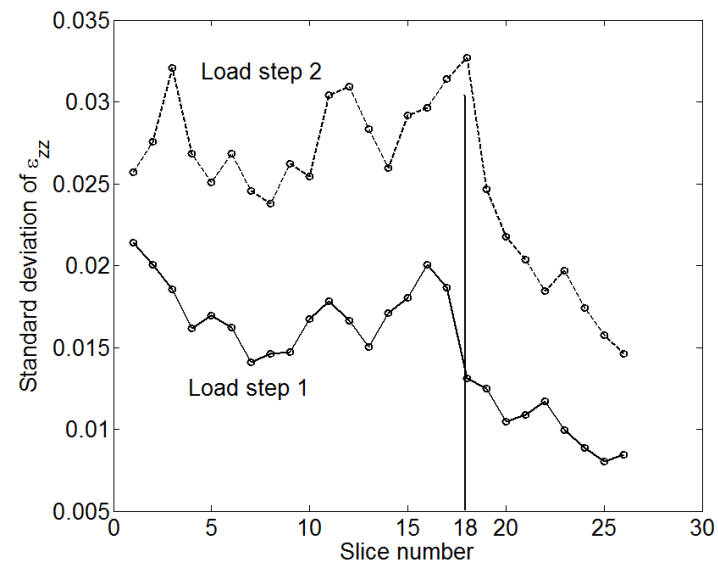

(в) $\varepsilon_{z z}$ for both load steps

Figure 14: Evolution of the standard deviation of the different strain components as a function of the $z$ position. Auxetic foam in tension. 


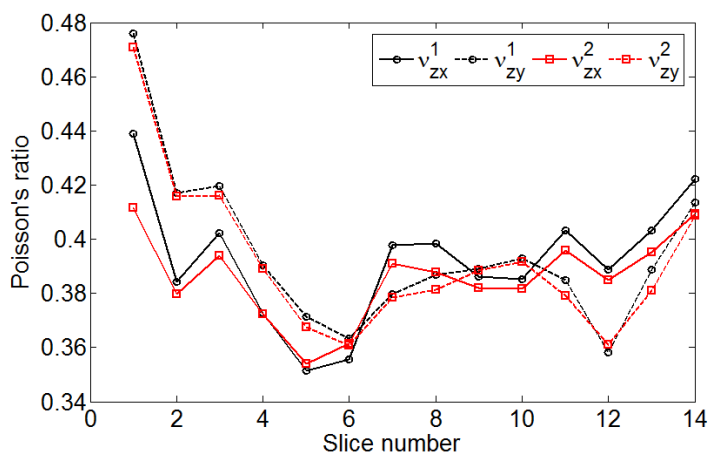

(A) Load step 1

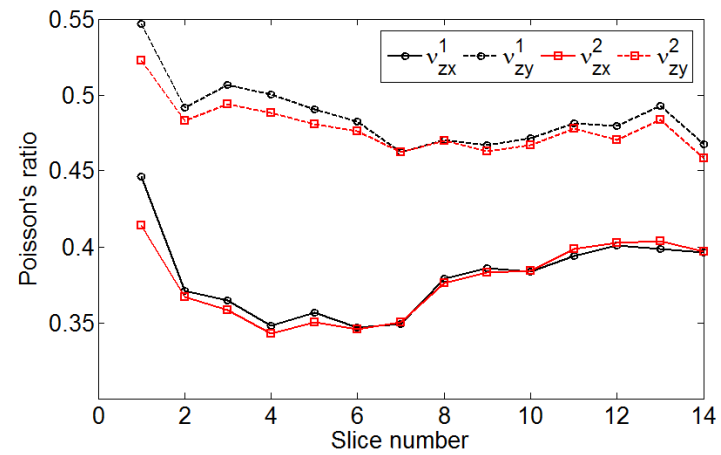

(B) Load step 2

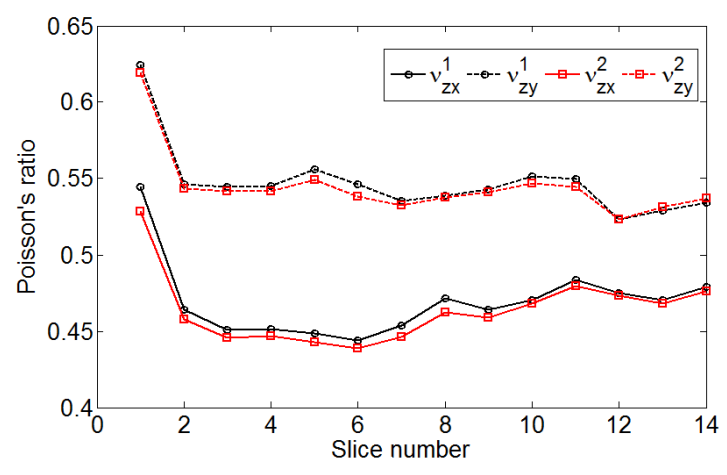

(C) Load step 3

Figure 15: Evolution of both Poisson's ratios calculated with the two methods of Section 6.1, for all three load steps. Standard foam in tension. 


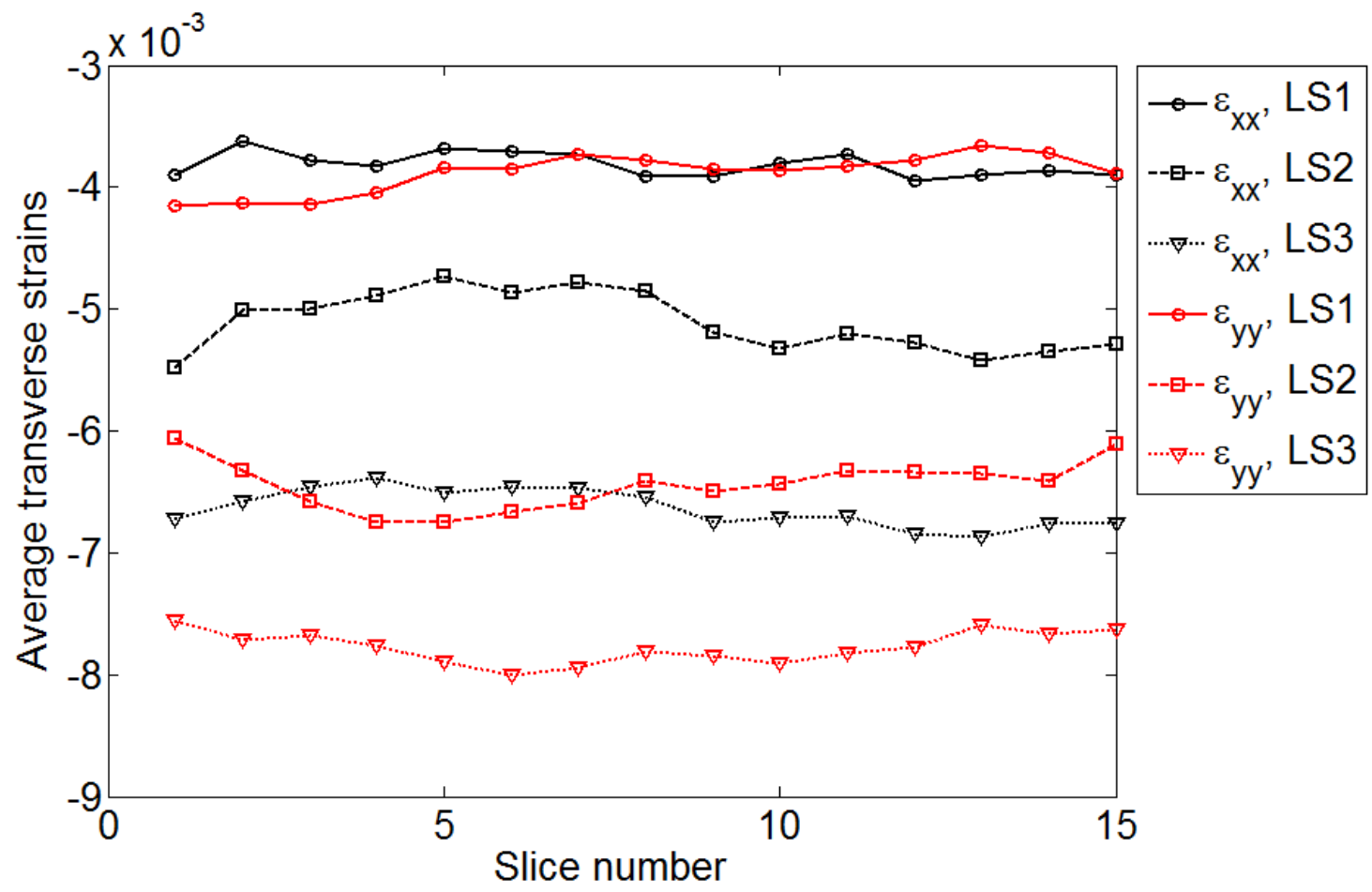

Figure 16: Evolution of the average transverse strains $\varepsilon_{x x}$ and $\varepsilon_{y y}$ as a function of $z$, for the three load steps, standard foam in tension. 


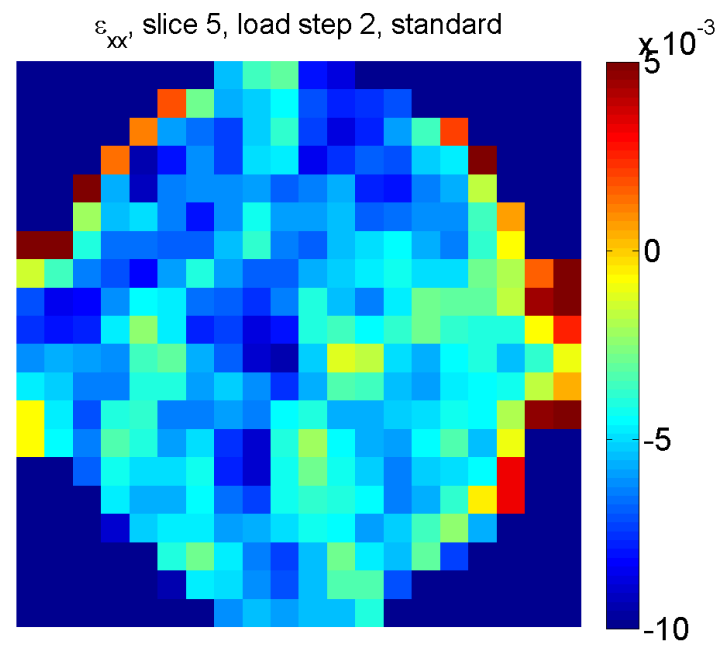

(A) $\varepsilon_{x x}$

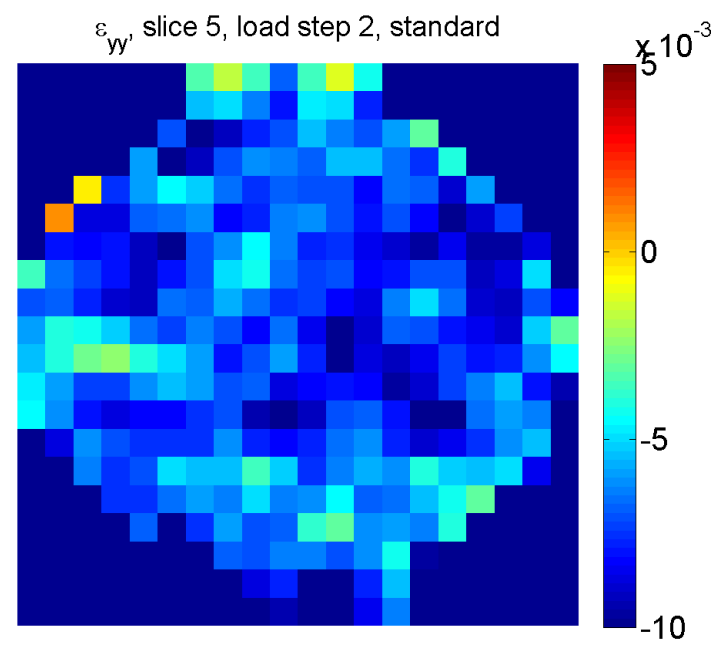

(B) $\varepsilon_{y y}$

$\varepsilon_{\mathrm{zz}}$, slice 5 , load step 2 , standard

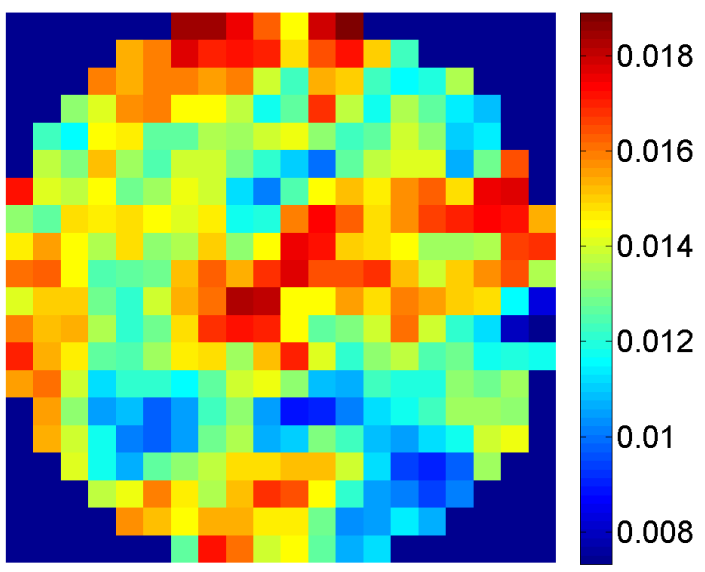

(C) $\varepsilon_{z z}$

Figure 17: Normal strain maps for slice 5, standard foam in tension, load step 2. 


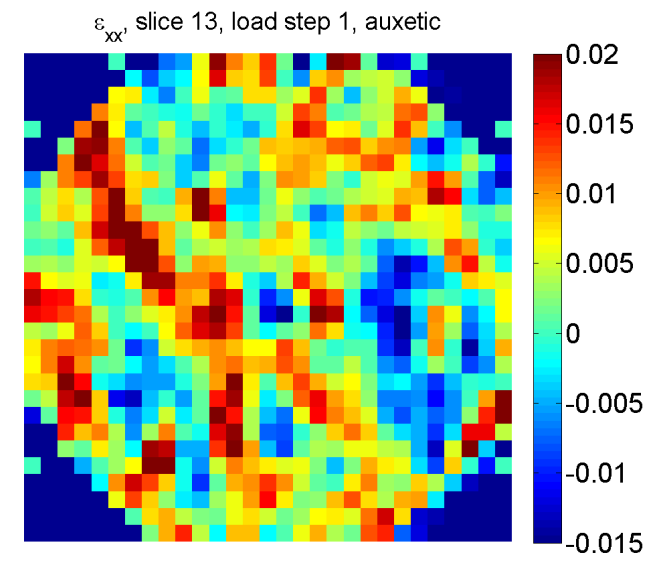

(A) $\varepsilon_{x x}$, slice 13 , load step 1

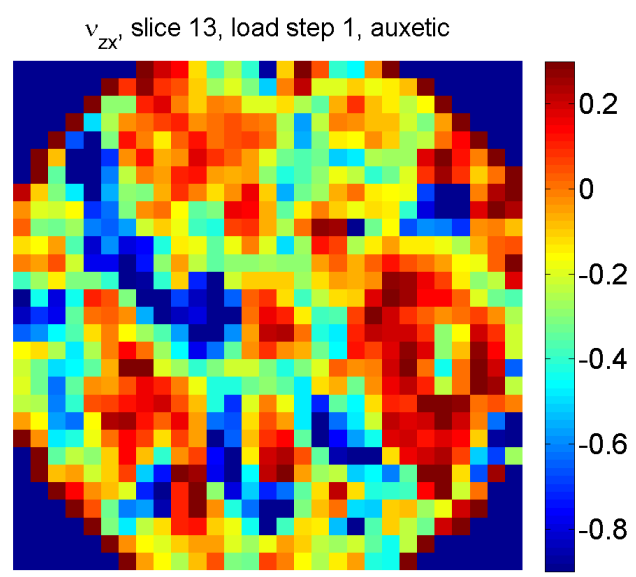

(c) $\nu_{z x}$, slice 13 , load step 1

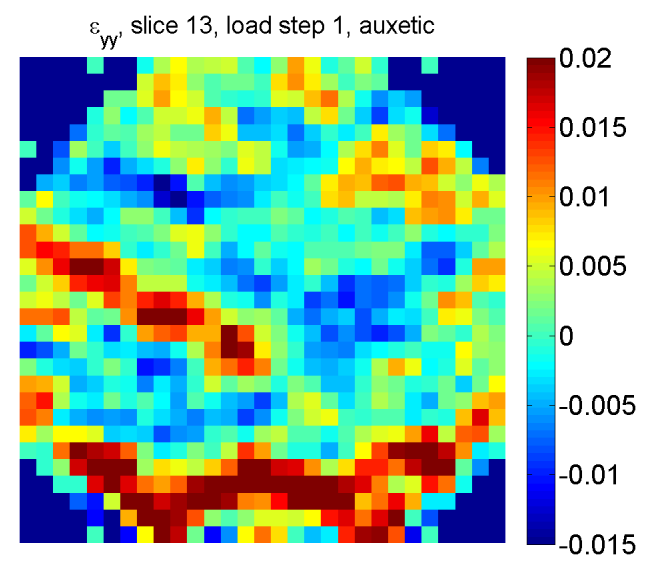

(B) $\varepsilon_{y y}$, slice 13 , load step 1

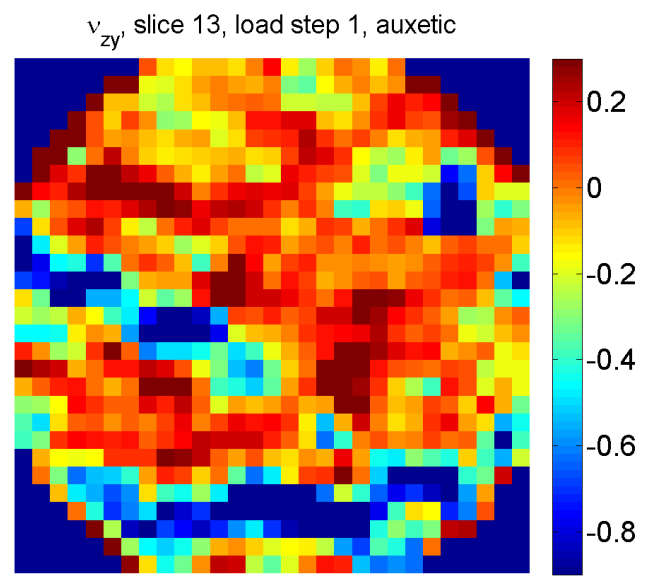

(D) $\nu_{z y}$, slice 13 , load step 1

Figure 18: Poisson's ratios and associated transverse strain maps, slice 13, load step 1, auxetic foam in tension. 


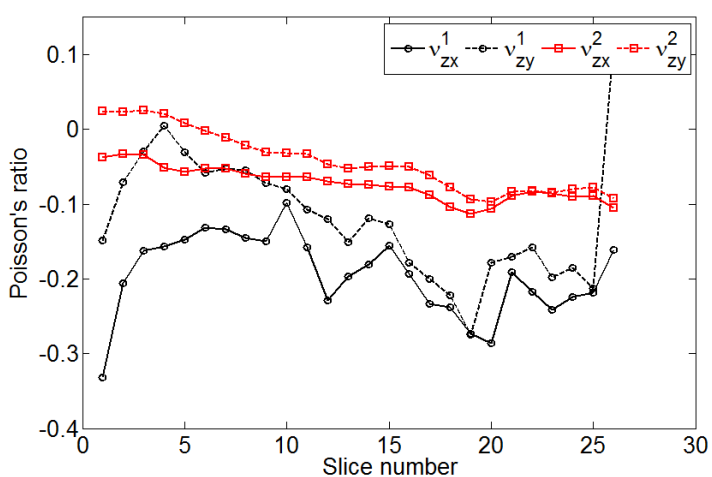

(A) Load step 1

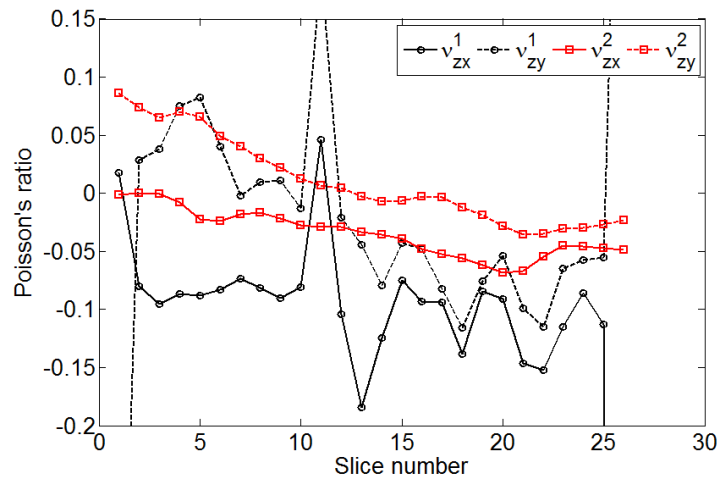

(B) Load step 2

Figure 19: Evolution of both Poisson's ratios calculated with the two methods of Section 6.1, for both load steps. Auxetic foam in tension. 